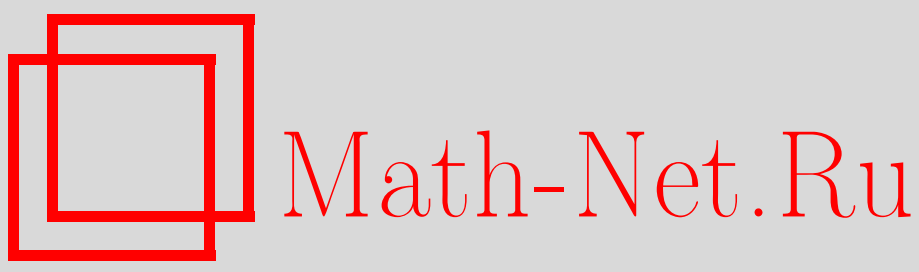

М. А. Королёв, Об аргументе дзета-функции Римана на критической прямой, Изв. РАН. Сер. матем., 2003, том 67, выпуск 2, 21-60

DOI: https://doi.org/10.4213/im426

Использование Общероссийского математического портала Math-Net.Ru подразумевает, что вы прочитали и согласны с пользовательским соглашением http://www . mathnet.ru/rus/agreement

Параметры загрузки:

IP : 52.87 .193 .239

26 апреля 2023 г., $12: 18: 15$ 
УДК 511

\author{
М. А. Королёв
}

\title{
Об аргументе дзета-функции Римана на критической прямой
}

\begin{abstract}
Доказана новая плотностная теорема для нулей дзета-функции Римана, лежащих в критической полосе. Утверждение теоремы применяется к задаче о числе перемен знака аргумента дзета-функции Римана на почти всех коротких промежутках критической прямой.

Библиография: 18 наименований.
\end{abstract}

\section{$\S 1$. Введение}

В настоящей работе доказывается оценка числа нулей $\zeta(s)$, лежаших в "узких" прямоугольниках вблизи критической прямой $\operatorname{Re} s=1 / 2$, а также рассматриваются приложения этой оценки к одной задаче из теории дзета-функции Римана.

В 1946 г. А. Сельберг [1] доказал следующее

УТВЕРЖДЕНИЕ 1. Пусть $\alpha$ - произвольное фиксированное число, $0<\alpha \leqslant$ $0.5, \quad T \geqslant T_{0}(\alpha)>0, \quad H=T^{0.5+\alpha}, \quad 0.5 \leqslant \sigma \leqslant 1$, и пусть $N(\sigma, T)$ обозначает число нулей $\zeta(s)$ в прямоугольнике $\sigma \leqslant \operatorname{Re} s<1,0<\operatorname{Im} s \leqslant T$. Тогда имеет место неравенство

$$
N(\sigma, T+H)-N(\sigma, T-H)=O\left(H T^{-0.25 \alpha(2 \sigma-1)} \ln T\right) .
$$

Одним из следствий этой плотностной теоремы является результат о количестве перемен знака функции $S(t)$, которая определяется следующим образом. Если $t>0$ отлично от ординаты нуля $\zeta(s)$, то

$$
S(t)=\frac{1}{\pi} \arg \zeta\left(\frac{1}{2}+i t\right)
$$

где $\arg \zeta(1 / 2+i t)$ получается путем непрерывного продолжения $\arg \zeta(s)$ вдоль ломаной линии, соединяющей точки $s=2(\arg \zeta(2)=0), s=2+i t$ и $s=1 / 2+i t$. Если же $t$ совпадает с ординатой какого-либо из нетривиальных нулей $\zeta(s)$, то полагают

$$
S(t)=\lim _{h \rightarrow 0} S(t+h)
$$

Е. Титчмаршем [2] (см. также [3, с. 222]) было установлено, что при $t>0$ функция $S(t)$ меняет свой знак бесконечное число раз. Более точное утверждение было получено А. Сельбергом в работе [1]. 
УТВЕРЖДЕНИЕ 2. Пусть $M(T)$ - число перемен знака $S(t)$ на промежутке $(0, T]$. Если величины $\alpha, T$ и Н удовлетворяют условиям утверждения 1, то существует постоянная $c=c(\alpha)>0$ такая, что

$$
M(T+H)-M(T) \geqslant H(\ln T)^{1 / 3} \exp (-c \sqrt{\ln \ln T}) .
$$

Улучшение последнего результата возможно по двум направлениям. Первое связано с заменой правой части неравенства большей величиной. Так, в 1981 г. А. Гош [4] показал, что при выполнении условий предыдущей теоремы справедливо более сильное неравенство

$$
M(T+H)-M(T) \geqslant H(\ln T) \exp \left(-\frac{c \ln \ln T}{(\ln \ln \ln T)^{0.5-\delta}}\right),
$$

где $\delta$ - сколь угодно малое фиксированное число, $0<\delta<0.5$, а $c=c(\alpha, \delta)>0-$ некоторая постоянная.

Второе направление связано с получением нижних оценок разности $M(T+H)-$ $M(T)$ для возможно меньших по сравнению с $T$ значений $H$. Нетривиальная оценка тригонометрической суммы специального вида позволила в 1996 г. А. А. Карацубе [5], [6] доказать плотностную теорему, подобную приведенной вьше, для $H=$ $T^{27 / 82+\alpha}$, где $\alpha$ - произвольное фиксированное число с условием $0<\alpha<0.001$. Следствием этого явилась оценка разности $M(T+H)-M(T)$ для указанных значений $H$ :

$$
M(T+H)-M(T) \geqslant H(\ln T)^{1 / 3} \exp \left(-c_{1} \sqrt{\ln \ln T}\right) .
$$

Использование идей и методов работ [1], [4]-[6] дало возможность автору несколько улучшить два последних результата и доказать, что для $H=T^{27 / 82+\alpha}$, $0<\alpha<0.001$, имеет место оценка

$$
M(T+H)-M(T) \geqslant H(\ln T) \exp \left(-\frac{c_{2} \ln \ln T}{\sqrt{\ln \ln \ln T}}\right),
$$

где $c_{2}=c_{2}(\alpha)>0$ (см. [7]).

В работе [8] упомянут следуюший результат, принадлежащий А. Сельбергу: если гипотеза Римана верна, то при любых фиксированных $\varepsilon, \varepsilon_{1}, 0<\varepsilon, \varepsilon_{1}<1$, для $T \geqslant T_{0}\left(\varepsilon, \varepsilon_{1}\right)>0$ и $H=T^{\varepsilon}$ выполняется неравенство

$$
M(T+H)-M(T) \geqslant H(\ln T)^{1-\varepsilon_{1}} .
$$

Множитель $(\ln T)^{-\varepsilon_{1}}$ в последнем неравенстве был заменен А. Гошем в [4] на величину

$$
\exp \left(-\frac{c \ln \ln T}{(\ln \ln \ln T)^{0.5-\delta}}\right)
$$

где $0<\delta<0.5$ и $c=c(\varepsilon, \delta)>0$. Доказательство этого утверждения для $H=T^{\varepsilon}$ также использует гипотезу Римана. Неравенства А. Сельберга и А. Гоша близки к предполагаемому окончательному результату

$$
M(T+H)-M(T) \sim \frac{H \ln T}{\sqrt{\pi \ln \ln T}}, \quad T^{\varepsilon} \leqslant H \leqslant T
$$

(гипотеза А. Сельберга; см. [9]).

Цель настоящей работы состоит в доказательстве двух основных утверждений: теорем А и В. Для их формулировки нам понадобятся следующие обозначения: $\varepsilon-$ произвольная постоянная, удовлетворяюшая неравенству $0<\varepsilon<0.01 ; X \geqslant$ $X_{0}(\varepsilon)>0 ; H=X^{\varepsilon}$. 
Teорема А. Пусть $c_{3}=c_{3}(\varepsilon)>0-$ постоянная, и пусть $\mathscr{E}_{1}-$ множество значений $T$ из промежутка $(X, 2 X]$, для которых при любом $\sigma, 0.5 \leqslant \sigma \leqslant 1$, верна оценка

$$
N(\sigma, T+H)-N(\sigma, T-H) \leqslant c_{3} H X^{-0.01 \varepsilon(2 \sigma-1)} \ln X .
$$

Тогда для меры әтого мноэсества справедлива асимптотическая формула

$$
\mu\left(\mathscr{E}_{1}\right)=X-O\left(X^{1-0.1 \varepsilon}\right) .
$$

Tеорема В. Существует постоянная $c_{4}=c_{4}(\varepsilon)>0$ такая, ито при всех Т из множества $\mathscr{E}_{1}$ справедливо неравенство

$$
M(T+H)-M(T) \geqslant H(\ln T) \exp \left(-\frac{c_{4} \ln \ln T}{\sqrt{\ln \ln \ln T}}\right) .
$$

Отметим, что доказательство первой теоремы основано на приемах, примененных А. А. Карацубой в [10].

Наряду с определенными выше $\varepsilon, X, H$ в работе используются следуюшие обозначения: $L=\ln X, k=[L] \geqslant 2, x=H^{0.01}, \Delta=H^{-1} L^{2}, H_{1}=H L^{-1}$, $P=\sqrt{T /(2 \pi)}, D=\left(2 H_{1}\right)^{k}$. Под символом $\sum_{A}^{B}$ понимается сумма $O(B)$ слагаемых, зависяших от параметра $A$. Буквы $c, c_{1}, c_{2}, \ldots$ обозначают положительные постоянные, зависящие от $\varepsilon$ и в разных соотношениях, вообще говоря, разные. Постоянные в знаках $O$ и « также зависят от $\varepsilon$. Через $\theta$ обозначены комплексные числа, по абсолютной величине не превосходящие единицы.

Моментом степени $\lambda \geqslant 0$ функции $f(u)$, определенной на промежутке $(a, b]$, будем называть интеграл

$$
J(f ; \lambda)=\int_{a}^{b}|f(u)|^{\lambda} d u .
$$

Если $\lambda=2 k$, где $k=1,2,3, \ldots$, то интегралы $J(f ; \lambda)$ будем называть также иетными моментами $f$. Кроме того, будем пользоваться стандартным обозначением

$$
S_{1}(t)=\int_{0}^{t} S(u) d u
$$

\section{§ 2. Вспомогательные утверждения}

При формулировке и доказательстве утверждений этого параграфа нам понадобится ряд новых обозначений: $\lambda_{1}, \lambda_{2}$ - целые числа, $1 \leqslant \lambda_{1}, \lambda_{2}<x ; \gamma=\lambda_{1} \lambda_{2}^{-1}$; $\left(2 \lambda_{1} \Delta\right)^{-1}<Y \leqslant \sqrt{X x} ; Y<Y_{1} \leqslant 2 Y ; Y_{2}=Y_{1}(1+\Delta)$. Для целых $m$ и $n$ положим

$$
\Phi(m, n ; \gamma)= \begin{cases}1, & \text { если } m \gamma<n \leqslant m \gamma(1+\Delta), \\ 0 & \text { в противном случае. }\end{cases}
$$

ЛЕмма 1. Пусть $a>0, \quad b$ - произвольное вещественное число. Тогда справедливо равенство

$$
\int_{-\infty}^{+\infty} \exp \left(-\left(\frac{t}{a}\right)^{2}+i b t\right) d t=\sqrt{\pi} a \exp \left(-\left(\frac{a b}{2}\right)^{2}\right) .
$$

Это утверждение достаточно известно. 
ЛЕмма 2. Для величины $R$, равной числу решений неравенства

$$
\left|y_{1} z_{2}-y_{2} z_{1}\right| \leqslant 24 \gamma Y^{2} X^{-1} L
$$

с дополнительными условиями

$$
Y<y_{r} \leqslant Y_{1}, \quad y_{r} \gamma<z_{r} \leqslant y_{r} \gamma(1+\Delta), \quad r=1,2
$$

справедлива оченка

$$
R \ll Y^{2} x^{6} H^{-1}
$$

ДокАЗАТЕЛЬСтво. Положим $z_{1} \lambda_{2}=y_{1} \lambda_{1}+h_{1}, z_{2} \lambda_{2}=y_{2} \lambda_{1}+h_{2}$. Определенные таким образом целые числа $h_{1}$ и $h_{2}$ удовлетворяют неравенствам

$$
1 \leqslant h_{r} \leqslant y_{r} \lambda_{1} \Delta \leqslant 2 Y x \Delta, \quad r=1,2 .
$$

Используя новые обозначения, получаем

$$
\begin{aligned}
y_{1} z_{2}-y_{2} z_{1} & =\lambda_{2}^{-1}\left(y_{1}\left(z_{2} \lambda_{2}\right)-y_{2}\left(z_{1} \lambda_{2}\right)\right) \\
& =\lambda_{2}^{-1}\left(y_{1}\left(y_{2} \lambda_{1}+h_{2}\right)-y_{2}\left(y_{1} \lambda_{1}+h_{1}\right)\right)=\lambda_{2}^{-1}\left(y_{1} h_{2}-y_{2} h_{1}\right) .
\end{aligned}
$$

Поскольку $\lambda_{2} \cdot 24 \gamma Y^{2} X^{-1} L \leqslant 24 x^{2} L \leqslant x^{3}$, то величина $R$ не превосходит числа решений неравенства $\left|y_{1} h_{2}-y_{2} h_{1}\right| \leqslant x^{3}$ с дополнительными условиями

$$
Y<y_{1}, y_{2} \leqslant 2 Y, \quad 1 \leqslant h_{1}, h_{2} \leqslant 2 Y x \Delta .
$$

При этих же ограничениях на переменные обозначим символом $r(m)$ количество решений уравнения $y_{1} h_{2}-y_{2} h_{1}=m$. Замечая, что

$$
r(m)=\int_{0}^{1}\left|\sum_{Y<y \leqslant 2 Y} \sum_{1 \leqslant h \leqslant 2 Y x \Delta} e^{2 \pi i \alpha y h}\right|^{2} e^{-2 \pi i \alpha m} d \alpha \leqslant r(0),
$$

получим цепочку неравенств

$$
R \leqslant \sum_{|m| \leqslant x^{3}} r(m) \leqslant\left(2 x^{3}+1\right) r(0) .
$$

Величина $r(0)$ равна числу решений симметричного уравнения $y_{1} h_{2}=y_{2} h_{1}$. Его левая часть может принимать не более $2 Y x \Delta(Y+1)$ значений. Фиксируя одно из них и обозначая его буквой $\nu$, приходим к уравнению $y_{1} h_{2}=\nu$, число решений которого не превосходит $\tau(\nu)$. Поскольку $Y<\nu \leqslant 2 Y \cdot 2 Y x \Delta<X$, то $\tau(\nu) \leqslant x$ при $X \geqslant X_{0}(\varepsilon)$. Заключаем, что

$$
r(0) \leqslant 2 Y x \Delta(Y+1) x \ll(Y x)^{2} \Delta, \quad R \ll x^{3}(Y x)^{2} \Delta \ll Y^{2} x^{6} H^{-1} .
$$

Лемма доказана. 
Лемма 3. Пусть $Y<u \leqslant Y_{1}, \quad Y \gamma<v \leqslant Y_{2} \gamma, u$ nycmb

$$
F(T)=\sum_{Y<m \leqslant u} \sum_{Y \gamma<n \leqslant v} \varepsilon_{1}(m) \varepsilon_{2}(n) \Phi(m, n ; \gamma)\left(\frac{m}{n}\right)^{i T}
$$

где $\left|\varepsilon_{1}(m)\right|=\left|\varepsilon_{2}(n)\right|=1$. Тогда для интеграла

$$
I=\int_{X}^{2 X}|F(T)| d T
$$

справедливо неравенство $I \ll X Y x^{3} H^{-0.5}$.

ДокАЗАТЕЛЬСТво. Применяя к интегралу $I$ неравенство Коши, получим, что $I^{2} \leqslant X J$

$$
J=\int_{X}^{2 X}|F(T)|^{2} d T .
$$

Поскольку $e^{4-(T / X)^{2}} \geqslant 1$ при $X \leqslant T \leqslant 2 X$, то

$$
\begin{aligned}
J \leqslant & e^{4} \int_{X}^{2 X} e^{-\left(\frac{T}{X}\right)^{2}}|F(T)|^{2} d T \leqslant e^{4} \int_{-\infty}^{+\infty} e^{-\left(\frac{T}{X}\right)^{2}}|F(T)|^{2} d T \\
= & e^{4} \sum_{Y<m_{1}, m_{2} \leqslant u} \sum_{Y \gamma<n_{1}, n_{2} \leqslant v} \varepsilon_{1}\left(m_{1}\right) \varepsilon_{2}\left(n_{1}\right) \bar{\varepsilon}_{1}\left(m_{2}\right) \bar{\varepsilon}_{2}\left(n_{2}\right) \Phi\left(m_{1}, n_{1} ; \gamma\right) \Phi\left(m_{2}, n_{2} ; \gamma\right) \\
& \times \int_{-\infty}^{+\infty} \exp \left(-\left(\frac{T}{X}\right)^{2}+i T \ln \frac{m_{1} n_{2}}{m_{2} n_{1}}\right) d T .
\end{aligned}
$$

Воспользовавшись формулой леммы 1, заключаем, что

$$
\begin{aligned}
J \leqslant & e^{4} \sqrt{\pi} X \sum_{Y<m_{1}, m_{2} \leqslant u} \sum_{Y \gamma<n_{1}, n_{2} \leqslant v} \Phi\left(m_{1}, n_{1} ; \gamma\right) \\
& \times \Phi\left(m_{2}, n_{2} ; \gamma\right) \exp \left(-\left(\frac{X}{2} \ln \frac{m_{1} n_{2}}{m_{2} n_{1}}\right)^{2}\right) \\
\ll & \sum_{Y<m_{1}, m_{2} \leqslant u} \sum_{\substack{m_{1} \gamma<n_{1} \leqslant m_{1} \gamma(1+\Delta) \\
m_{2} \gamma<n_{2} \leqslant m_{2} \gamma(1+\Delta)}} \exp \left(-\left(\frac{X}{2} \ln \frac{m_{1} n_{2}}{m_{2} n_{1}}\right)^{2}\right) .
\end{aligned}
$$

Последнюю сумму разобьем на две: $\sigma_{1}$ и $\sigma_{2}$. К сумме $\sigma_{1}$ отнесем все слагаемые, для которых

$$
\left|m_{1} n_{2}-m_{2} n_{1}\right| \leqslant 24 \gamma Y^{2} X^{-1} L,
$$

а к $\sigma_{2}$ - все остальные. Оценивая каждое слагаемое первой суммы тривиально, заключаем, что $\sigma_{1}$ не превосходит числа решений неравенства

$$
\left|m_{1} n_{2}-m_{2} n_{1}\right| \leqslant 24 \gamma Y^{2} X^{-1} L
$$

с дополнительными условиями

$$
Y<m_{r} \leqslant 2 Y, \quad m_{r} \gamma<n_{r} \leqslant m_{r} \gamma(1+\Delta), \quad r=1,2 .
$$


Согласно лемме $2 \sigma_{1} \ll Y^{2} x^{6} H^{-1}$. Пусть теперь $m_{1}, m_{2}, n_{1}, n_{2}$ таковы, что

$$
\left|m_{1} n_{2}-m_{2} n_{1}\right|>24 \gamma Y^{2} X^{-1} L .
$$

Тогда в случае $m_{1} n_{2}>m_{2} n_{1}$ получим цепочку неравенств

$$
\begin{aligned}
\ln \frac{m_{1} n_{2}}{m_{2} n_{1}} & =\ln \left(1+\frac{m_{1} n_{2}-m_{2} n_{1}}{m_{2} n_{1}}\right)>\ln \left(1+\frac{24 \gamma Y^{2} X^{-1} L}{2 Y \cdot 3 Y \gamma}\right) \\
& =\ln \left(1+4 L X^{-1}\right) \geqslant 2 L X^{-1},
\end{aligned}
$$

откуда следует, что

$$
\exp \left(-\left(\frac{X}{2} \ln \frac{m_{1} n_{2}}{m_{2} n_{1}}\right)^{2}\right) \leqslant \exp \left(-\left(\frac{X}{2} \frac{2 L}{X}\right)^{2}\right)=e^{-L^{2}}
$$

Это же неравенство будет выполняться и в случае $m_{1} n_{2}<m_{2} n_{1}$. Поскольку число слагаемых в $\sigma_{2}$ не превосходит $Y^{4}$, то $\sigma_{2} \leqslant Y^{4} e^{-L^{2}} \ll Y^{2} x^{6} H^{-1}$. Таким образом,

$$
J \ll X Y^{2} x^{6} H^{-1} \text { и } I \ll X Y x^{3} H^{-0.5} .
$$

Лемма доказана.

ЛЕмма 4. Пусть $Q=[P \sqrt{\gamma}], 0<u \leqslant \Delta \sqrt{X \gamma}$, u nycmb

$$
F_{1}(T)=\sum_{0 \leqslant \nu \leqslant u} \varepsilon(\nu)(Q-\nu)^{i T}
$$

где $|\varepsilon(\nu)|=1$. Тогда для интеграла

$$
I_{1}=\int_{X}^{2 X}\left|F_{1}(T)\right|^{2} d T
$$

справедливо неравенство

$$
I_{1} \ll(x \sqrt{X})^{3} H^{-1} .
$$

ДокАЗАТЕЛЬСТво. Определим целые числа $n_{0}$ и $n_{1}$ так, чтобы выполнялись неравенства

$$
\begin{aligned}
& 2 \pi \gamma^{-1} n_{0}^{2} \leqslant X<2 \pi \gamma^{-1}\left(n_{0}+1\right)^{2} \\
& 2 \pi \gamma^{-1} n_{1}^{2} \leqslant 2 X<2 \pi \gamma^{-1}\left(n_{1}+1\right)^{2}
\end{aligned}
$$

Тогда

$$
I_{1} \leqslant \int_{2 \pi \gamma^{-1} n_{0}^{2}}^{2 \pi \gamma^{-1}\left(n_{1}+1\right)^{2}}\left|F_{1}(T)\right|^{2} d T=\sum_{n=n_{0}}^{n_{1}} I(n),
$$

где

$$
I(n)=\int_{2 \pi \gamma^{-1} n^{2}}^{2 \pi \gamma^{-1}(n+1)^{2}}\left|F_{1}(T)\right|^{2} d T .
$$


Зафиксируем произвольным образом $n, n_{0} \leqslant n \leqslant n_{1}$. Если $2 \pi \gamma^{-1} n^{2} \leqslant T<$ $2 \pi \gamma^{-1}(n+1)^{2}$, то $n \leqslant P \sqrt{\gamma}=\sqrt{T \gamma /(2 \pi)}<n+1, Q=[P \sqrt{\gamma}]=n$, и сумма $F_{1}(T)$ принимает следующий вид:

$$
F_{1}(T)=\sum_{0 \leqslant \nu \leqslant u} \varepsilon(\nu)(n-\nu)^{i T}
$$

Поэтому

$$
\begin{aligned}
I(n)= & 2 \pi \gamma^{-1}([u]+1)\left((n+1)^{2}-n^{2}\right) \\
& +\sum_{\substack{0 \leqslant \nu_{1}, \nu_{2} \leqslant u \\
\nu_{1} \neq \nu_{2}}} \varepsilon\left(\nu_{1}\right) \bar{\varepsilon}\left(\nu_{2}\right) \int_{2 \pi \gamma^{-1} n^{2}}^{2 \pi \gamma^{-1}(n+1)^{2}}\left(\frac{n-\nu_{1}}{n-\nu_{2}}\right)^{i T} d T .
\end{aligned}
$$

Интегрируя и переходя к неравенствам, получим

$$
|I(n)| \ll \gamma^{-1} n \Delta \sqrt{X \gamma}+\sum_{0 \leqslant \nu_{1}<\nu_{2} \leqslant u}\left(\ln \left(\frac{n-\nu_{1}}{n-\nu_{2}}\right)\right)^{-1} .
$$

Если $\nu_{2}>\nu_{1}$ то

$$
\begin{aligned}
\ln \left(\frac{n-\nu_{1}}{n-\nu_{2}}\right) & =\ln \left(\frac{1-\nu_{1} / n}{1-\nu_{2} / n}\right)=\sum_{r=1}^{+\infty} \frac{\nu_{2}^{r}-\nu_{1}^{r}}{r n^{r}} \\
& =\frac{\nu_{2}-\nu_{1}}{n}\left(1+\sum_{r=2}^{+\infty} \frac{\nu_{2}^{r}-\nu_{1}^{r}}{\nu_{2}-\nu_{1}} \frac{1}{r n^{r-1}}\right)>\frac{\nu_{2}-\nu_{1}}{n}
\end{aligned}
$$

так что

$$
\begin{aligned}
|I(n)| & \ll \gamma^{-1} n \Delta \sqrt{X \gamma}+\sum_{0 \leqslant \nu_{1}<\nu_{2} \leqslant u} \frac{n}{\nu_{2}-\nu_{1}} \\
& \ll n\left(\gamma^{-1} \Delta \sqrt{X \gamma}+\sum_{0 \leqslant \nu_{1} \leqslant u} \sum_{1 \leqslant r \leqslant u} r^{-1}\right) \ll n \Delta \sqrt{X \gamma}\left(\gamma^{-1}+L\right) \\
& \ll n x \Delta \sqrt{X \gamma} \ll n \Delta \sqrt{X} x^{1.5} .
\end{aligned}
$$

Переходя к оценке $I_{1}$, будем иметь

$$
\begin{aligned}
I_{1} & \ll \Delta \sqrt{X} x^{1.5} \sum_{n=n_{0}}^{n_{1}} n \ll \Delta \sqrt{X} x^{1.5} n_{1}^{2} \ll \Delta \sqrt{X} x^{1.5} X \gamma \\
& \ll X^{1.5} x^{2.5} L^{2} H^{-1} \ll(x \sqrt{X})^{3} H^{-1} .
\end{aligned}
$$

Лемма доказана. 


\section{§ 3. Основные леммы}

В этом параграфе доказажем две леммы, которые являются основными при доказательстве плотностной теоремы (теоремы А).

ЛЕмма 5. Пусть ү пробегает все рачиональные дроби, иислитель и знаменатель которых не превосходят $x$, и пусть

$$
r\left(\sigma_{1}, T\right)=\sum_{\gamma} \int_{-2 H}^{2 H} \int_{-H_{1}}^{H_{1}} \ldots \int_{-H_{1}}^{H_{1}}\left|W\left(\sigma_{1}, T_{1} ; \gamma\right)\right| d t d t_{1} \ldots d t_{k},
$$

əде

$$
W\left(\sigma_{1}, T_{1} ; \gamma\right)=\sum_{m \leqslant P_{1}} \sum_{n \leqslant P_{2}}(m n)^{-\sigma_{1}} \Phi(m, n ; \gamma)\left(\frac{m}{n}\right)^{i T_{1}}
$$

$P_{1}=P / \sqrt{\gamma}, \quad P_{2}=P \sqrt{\gamma}, \quad T_{1}=T+t+t_{1}+\cdots+t_{k}$. Tогда существует множество $E$ такое, что $\mu(E) \ll X H^{-0.1}$, и для всех $T$ из промежутка $(X, 2 X]$, не принадлежащих $E$, при любом $\sigma_{1}$, удовлетворяющем условию $0 \leqslant \sigma_{1} \leqslant 1$, справедлива оченка

$$
r\left(\sigma_{1}, T\right) \ll H D\left(1+X^{0.5-\sigma_{1}}\right) H^{-0.3} .
$$

ДокАЗАТЕЛЬСтво. Зафиксируем произвольное значение $\gamma=\lambda_{1} \lambda_{2}^{-1}$ и преобразуем сумму $W_{1}=W\left(\sigma_{1}, T_{1} ; \gamma\right)$ так, чтобы границы изменения $m$ и $n$ в ней не зависели от $T$. Пусть $A=[\sqrt{X x / \pi}]+2$. Положим

$$
\begin{aligned}
& \delta_{1}(m)=(2 A+1)^{-1} \sum_{a=-A}^{A} \sum_{m_{1} \leqslant P_{1}} \exp \left(2 \pi i \frac{a\left(m-m_{1}\right)}{2 A+1}\right), \\
& \delta_{2}(n)=(2 A+1)^{-1} \sum_{b=-A}^{A} \sum_{n_{1} \leqslant P_{2}} \exp \left(2 \pi i \frac{b\left(n-n_{1}\right)}{2 A+1}\right) .
\end{aligned}
$$

Поскольку $P_{1}, P_{2}<A$, то при $r=1,2$

$$
\delta_{r}(\nu)= \begin{cases}1, & \text { если } 1 \leqslant \nu \leqslant P_{r}, \\ 0 & \text { в противном случае. }\end{cases}
$$

Используя введенные функции $\delta_{1}(m)$ и $\delta_{2}(n)$, запишем $W_{1}$ в следуюшем виде:

$$
W_{1}=\sum_{m, n \leqslant A} \delta_{1}(m) \delta_{2}(n)(m n)^{-\sigma_{1}} \Phi(m, n ; \gamma)\left(\frac{m}{n}\right)^{i T_{1}} .
$$

Подставляя в это равенство явные выражения для $\delta_{r}(\nu), r=1,2$, и меняя местами порядки суммирования, получим

$$
W_{1}=(2 A+1)^{-2} \sum_{a, b=-A}^{A}\left(\sum_{m_{1} \leqslant P_{1}} e^{-2 \pi i \frac{a m_{1}}{2 A+1}}\right)\left(\sum_{n_{1} \leqslant P_{2}} e^{-2 \pi i \frac{b n_{1}}{2 A+1}}\right) W_{2}(a, b),
$$


где

$$
W_{2}(a, b)=\sum_{m, n \leqslant A}(m n)^{-\sigma_{1}} e^{2 \pi i \frac{a m+b n}{2 A+1}} \Phi(m, n ; \gamma)\left(\frac{m}{n}\right)^{i T_{1}}
$$

Границы изменения $m$ и $n$ в каждой из сумм $W_{2}(a, b)$ уже не зависят от $T$. Переходя к неравенствам, получим

$$
\left|W_{1}\right| \leqslant \sum_{a, b=-A}^{A}(|a|+1)^{-1}(|b|+1)^{-1}\left|W_{2}(a, b)\right| .
$$

Зафиксируем произвольно значения $a$ и $b$ и преобразуем сумму $W_{2}=W_{2}(a, b)$. Промежуток изменения величины $m$ разобьем на $O(L)$ промежутков вида $M<$ $m \leqslant M_{1}$, где $M_{1} \leqslant 2 M$. Так как $\Phi(m, n ; \gamma)$ отлично от нуля лишь при $m \gamma<n \leqslant$ $m \gamma(1+\Delta)$, то при $M<m \leqslant M_{1}$ все значения $n$, для которых $\Phi(m, n ; \gamma) \neq 0$, содержатся в промежутке $M \gamma<n \leqslant M_{1} \gamma(1+\Delta)=M_{2} \gamma$. Перепишем теперь неравенство, которому удовлетворяют $m$ и $n$, в виде

$$
m \lambda_{1}<n \lambda_{2} \leqslant m \lambda_{1}(1+\Delta)
$$

Если $M<\left(2 \lambda_{1} \Delta\right)^{-1}$, то для каждого $m$ из промежутка $M<m \leqslant M_{1}$ будут выполняться неравенства

$$
m \lambda_{1}(1+\Delta) \leqslant m \lambda_{1}+2 M \lambda_{1} \Delta<m \lambda_{1}+2 \lambda_{1} \Delta\left(2 \lambda_{1} \Delta\right)^{-1}=m \lambda_{1}+1
$$

и сумма по $n$ оказывается пустой. Учитывая это замечание, представим $W_{2}$ в виде

$$
W_{2}=\sum_{M}^{L} W_{3}(M)
$$

где

$$
\begin{gathered}
W_{3}(M)=\sum_{M<m \leqslant M_{1}} \sum_{M \gamma<n \leqslant M_{2} \gamma}(m n)^{-\sigma_{1}} \varepsilon_{1}(m) \varepsilon_{2}(n) \Phi(m, n ; \gamma)\left(\frac{m}{n}\right)^{i T_{1}} \\
\varepsilon_{1}(m)=e^{2 \pi i \frac{a m}{2 A+1}} m^{i\left(t+t_{1}+\cdots+t_{k}\right)}, \quad \varepsilon_{2}(n)=e^{2 \pi i \frac{b n}{2 A+1}} n^{-i\left(t+t_{1}+\cdots+t_{k}\right)}
\end{gathered}
$$

а штрих означает суммирование по $M \geqslant\left(2 \lambda_{1} \Delta\right)^{-1}$. Зафиксируем любое значение $M$ и применим к сумме $W_{3}(M)$ формулу частного суммирования в следующей форме (см. [10]): если $g_{1}(u)$ и $g_{2}(v)$ - непрерывно дифференцируемые функции, $G(m, n)$ - произвольная комплекснозначная функция, то

$$
\begin{aligned}
& \sum_{A_{1}<m \leqslant B_{1}} \sum_{A_{2}<m \leqslant B_{2}} g_{1}(m) g_{2}(n) G(m, n)=\int_{A_{1}}^{B_{1}} \int_{A_{2}}^{B_{2}} F(u, v) g_{1}^{\prime}(u) g_{2}^{\prime}(v) d u d v \\
& \quad-g_{2}\left(B_{2}\right) \int_{A_{1}}^{B_{1}} F\left(u, B_{2}\right) g_{1}^{\prime}(u) d u-g_{1}\left(B_{1}\right) \int_{A_{2}}^{B_{2}} F\left(B_{1}, v\right) g_{2}^{\prime}(v) d v \\
& \quad+g_{1}\left(B_{1}\right) g_{2}\left(B_{2}\right) F\left(B_{1}, B_{2}\right),
\end{aligned}
$$


где

$$
F(u, v)=\sum_{A_{1}<m \leqslant u} \sum_{A_{2}<m \leqslant v} G(m, n) .
$$

Положим $g_{1}(u)=u^{-\sigma_{1}}, g_{2}(v)=v^{-\sigma_{1}}, A_{1}=M, B_{1}=M_{1}, A_{2}=M \gamma$, $B_{2}=M_{2} \gamma, G(m, n)=\varepsilon_{1}(m) \varepsilon_{2}(n) \Phi(m, n ; \gamma)\left(m n^{-1}\right)^{i T_{1}}$. Тогда

$$
\begin{aligned}
W_{3}= & \sigma_{1}^{2} \int_{M}^{M_{1}} \int_{M \gamma}^{M_{2} \gamma} F(u, v)(u v)^{-\sigma_{1}-1} d u d v \\
& +\sigma_{1}\left(M_{2} \gamma\right)^{-\sigma_{1}} \int_{M}^{M_{1}} F\left(u, M_{2} \gamma\right) u^{-\sigma_{1}-1} d u \\
& +\sigma_{1} M_{1}^{-\sigma_{1}} \int_{M \gamma}^{M_{2} \gamma} F\left(M_{1}, v\right) v^{-\sigma_{1}-1} d v \\
& +\left(M_{1} M_{2} \gamma\right)^{-\sigma_{1}} F\left(M_{1}, M_{2} \gamma\right) .
\end{aligned}
$$

Пользуясь тем, что $u \asymp M, v \asymp M \gamma, \gamma^{-\sigma_{1}} \ll x$, находим

$$
\begin{aligned}
\left|W_{3}\right| \ll & x M^{-2 \sigma_{1}}\left(\gamma^{-1} M^{-2} \int_{M}^{M_{1}} \int_{M \gamma}^{M_{2} \gamma}|F(u, v)| d u d v\right. \\
& +(\gamma M)^{-1}\left(\int_{M}^{M_{1}}\left|F\left(u, M_{2} \gamma\right)\right| d u+\int_{M \gamma}^{M_{2} \gamma}\left|F\left(M_{1}, v\right)\right| d v\right) \\
& \left.+\left|F\left(M_{1}, M_{2} \gamma\right)\right|\right) .
\end{aligned}
$$

Используя полученные оценки, будем иметь

$$
r\left(\sigma_{1}, T\right) \ll x \sum_{M}^{L} M^{-2 \sigma_{1}} K(M ; T),
$$

где

$$
\begin{aligned}
K(M ; T)= & \sum_{\gamma} \sum_{a, b=-A}^{A}(|a|+1)^{-1}(|b|+1)^{-1} \\
& \times \int_{-2 H}^{2 H} \int_{-H_{1}}^{H_{1}} \ldots \int_{-H_{1}}^{H_{1}}\left(\gamma^{-1} M^{-2} \int_{M}^{M_{1}} \int_{M \gamma}^{M_{2} \gamma}|F(u, v)| d u d v\right. \\
& +(\gamma M)^{-1}\left(\int_{M}^{M_{1}}\left|F\left(u, M_{2} \gamma\right)\right| d u+\int_{M \gamma}^{M_{2} \gamma}\left|F\left(M_{1}, v\right)\right| d v\right) \\
& \left.+\left|F\left(M_{1}, M_{2} \gamma\right)\right|\right) d t d t_{1} \ldots d t_{k} .
\end{aligned}
$$

Обозначим

$$
I(u, v)=\int_{X}^{2 X}|F(u, v)| d T
$$


Положив в лемме $3 Y=M, Y_{1}=M_{1}, Y_{2}=M_{2}$, заключаем, что

$$
I(u, v) \ll X M x^{3} H^{-0.5} .
$$

Воспользовавшись этой оценкой, находим

$$
\begin{aligned}
\int_{X}^{2 X} K(M ; T) d T \ll & \sum_{\gamma} \sum_{a, b=-A}^{A}(|a|+1)^{-1}(|b|+1)^{-1} H D X M x^{3} H^{-0.5} \\
& \times\left(\gamma^{-1} M^{-2} \gamma M^{2}+(\gamma M)^{-1}(M+M \gamma)+M\right) \\
& \ll H D X M x^{3} H^{-0.5} L^{2} \sum_{\gamma}\left(1+\gamma^{-1}\right) \ll H D X M x^{5} L^{3} H^{-0.5} .
\end{aligned}
$$

Обозначим символом $E(M)$ множество тех $T$ из промежутка $(X, 2 X]$, для которых выполнено неравенство

$$
K(M ; T)>H D M x^{5} L^{4} H^{-0.4},
$$

и оценим меру этого множества сверху. Для этого проинтегрируем по $E(M)$ обе части последнего неравенства и воспользуемся полученной оценкой среднего значения величины $K(M ; T)$ :

$$
\begin{aligned}
H D M x^{5} L^{4} H^{-0.4} \mu(E(M)) & \leqslant \int_{E(M)} K(M ; T) d T \leqslant \int_{X}^{2 X} K(M ; T) d T \\
& \ll H D X M x^{5} L^{3} H^{-0.5} .
\end{aligned}
$$

Производя сокращения, получим

$$
\mu(E(M)) \ll X H^{-0.1} L^{-1} .
$$

Построим такое множество $E(M)$ для каждого из $O(L)$ значений $M$ и обозначим символом $E$ их объединение. Тогда $\mu(E) \ll X H^{-0.1}$ и на дополнении к $E$ для каждого $M$ справедливо неравенство

$$
K(M ; T) \ll H D M x^{5} L^{3} H^{-0.4} .
$$

Для таких $T$ при любом значении $\sigma_{1}, 0 \leqslant \sigma_{1} \leqslant 1$, имеет место оценка

$$
r\left(\sigma_{1}, T\right) \ll x \sum_{M}^{L} H D M x^{5} L^{3} H^{-0.4} M^{-2 \sigma_{1}} \ll H D x^{6} L^{3} H^{-0.4} \sum_{M}^{L} M^{1-2 \sigma_{1}} .
$$

Поскольку $1 \leqslant(2 x \Delta)^{-1}<M \leqslant \sqrt{X x}$, то

$$
\begin{aligned}
r\left(\sigma_{1}, T\right) & \ll H D x^{6} L^{4} H^{-0.4}\left(1+(x X)^{0.5-\sigma_{1}}\right) \\
& \ll H D x^{7} H^{-0.4}\left(1+X^{0.5-\sigma_{1}}\right) \ll H D\left(1+X^{0.5-\sigma_{1}}\right) H^{-0.3} .
\end{aligned}
$$

Лемма доказана. 
ЛЕмма 6. Пусть $\gamma$ пробегает все рациональные дроби, числитель и знаменатель которых не превосходят $x$, и пусть

$$
\varrho(\sigma, T)=\sum_{\gamma} \int_{-2 H}^{2 H} \int_{-H_{1}}^{H_{1}} \cdots \int_{-H_{1}}^{H_{1}}\left|V\left(\sigma, T_{1} ; \gamma\right)\right| d t d t_{1} \ldots d t_{k},
$$

гдe

$$
V\left(\sigma, T_{1} ; \gamma\right)=\sum_{Q_{2}<m, n \leqslant Q_{1}} m^{\sigma-1} n^{-\sigma}(m n)^{i T_{1}},
$$

$Q_{1}=P \sqrt{\gamma}, Q_{2}=Q_{1}(1-\Delta)$. Тогда существует множество $G$ такое, ито $\mu(G) \ll X H^{-0.1}$, и для всех $T$ из промежутка $(X, 2 X]$, не принадлежащих $G$, при любом $\sigma$, удовлетворяющем условию $0.5 \leqslant \sigma \leqslant 1$, справедлива оченка

$$
\varrho(\sigma, T) \ll H D H^{-0.8} .
$$

ДокАЗАТЕльСтво. Зафиксируем произвольное значение $\gamma=\lambda_{1} \lambda_{2}^{-1}$ и преобразуем сумму $V_{1}=V\left(\sigma, T_{1} ; \gamma\right)$ так, чтобы границы изменения $m$ и $n$ не зависели от $T$. Положим $Q=\left[Q_{1}\right]+1, q=\left[Q_{1}\right]-\left[Q_{2}\right]-1, B=[\Delta \sqrt{X \gamma / \pi}]+2$. Используя эти обозначения, запишем сумму $V_{1}$ в виде

$$
V_{1}=\sum_{\mu, \nu=1}^{q}(Q-\mu)^{-\sigma+i T_{1}}(Q-\nu)^{\sigma-1+i T_{1}}
$$

Положим

$$
\begin{aligned}
& \delta_{1}(\mu)=(2 B+1)^{-1} \sum_{a=-B}^{B} \sum_{\mu_{1}=1}^{q} \exp \left(2 \pi i \frac{a\left(\mu-\mu_{1}\right)}{2 B+1}\right) \\
& \delta_{2}(\nu)=(2 B+1)^{-1} \sum_{b=-B}^{B} \sum_{\nu_{1}=1}^{q} \exp \left(2 \pi i \frac{b\left(\nu-\nu_{1}\right)}{2 B+1}\right) .
\end{aligned}
$$

Тогда для $r=1,2$

$$
\delta_{r}(\lambda)= \begin{cases}1, & \text { если } 1 \leqslant \lambda \leqslant q, \\ 0 & \text { в противном случае. }\end{cases}
$$

Теперь сумма $V_{1}$ принимает вид

$$
V_{1}=\sum_{\mu, \nu=1}^{B} \delta_{1}(\mu) \delta_{2}(\nu)(Q-\mu)^{-\sigma+i T_{1}}(Q-\nu)^{\sigma-1+i T_{1}}
$$

Подставляя явные выражения для $\delta_{1}(\mu)$ и $\delta_{2}(\nu)$ и меняя местами порядки суммирования, получим

$$
V_{1}=(2 B+1)^{-2} \sum_{a, b=1}^{B}\left(\sum_{\mu_{1}=1}^{q} e^{-2 \pi i \frac{a \mu_{1}}{2 B+1}}\right)\left(\sum_{\nu_{1}=1}^{q} e^{-2 \pi i \frac{b \nu_{1}}{2 B+1}}\right) V_{2}(a, b),
$$


где

$$
V_{2}(a, b)=\sum_{\mu, \nu=1}^{B} \delta_{1}(\mu) \delta_{2}(\nu)(Q-\mu)^{-\sigma+i T_{1}}(Q-\nu)^{\sigma-1+i T_{1}} e^{2 \pi i \frac{a \mu+b \nu}{2 B+1}}
$$

Переходя к неравенствам, находим

$$
\left|V_{1}\right| \leqslant \sum_{a, b=1}^{B}(|a|+1)^{-1}(|b|+1)^{-1}\left|V_{2}(a, b)\right|
$$

Каждую из сумм $V_{2}(a, b)$ можно представить в виде $V_{2}(a, b)=V_{3}(a) V_{3}(b)$,

$$
V_{3}(c)=\sum_{\nu=1}^{B}(Q-\nu)^{-\sigma_{1}} e^{2 \pi i \frac{c \nu}{2 B+1}}
$$

а $\sigma_{1}$ равно $\sigma$ или $1-\sigma$. Применяя к сумме $V_{3}(c)$ формулу частного суммирования, получаем

$$
V_{3}(c)=(Q-B)^{-\sigma_{1}} F(B ; c)+\sigma_{1} \int_{0}^{B}(Q-u)^{-\sigma_{1}-1} F(u ; c) d u
$$

где

$$
F(u ; c)=\sum_{0<\nu \leqslant u}(Q-\nu)^{i T_{1}} e^{2 \pi i \frac{c \nu}{2 B+1}}
$$

Переходя к оценкам и пользуясь тем, что $Q-u \asymp Q \asymp \sqrt{X \gamma}$, приходим к неравенству

$$
\left|V_{3}(c)\right| \ll Q^{-\sigma_{1}}\left(|F(B ; c)|+Q^{-1} \int_{0}^{B}|F(u ; c)| d u\right) .
$$

Полагая в последнем неравенстве $\sigma_{1}=\sigma, c=a$ и $\sigma_{1}=1-\sigma, c=b$, находим

$$
\begin{aligned}
\left|V_{2}(a, b)\right| \ll & Q^{-1}\left(|F(B ; a)|+Q^{-1} \int_{0}^{B}|F(u ; a)| d u\right) \\
& \times\left(|F(B ; b)|+Q^{-1} \int_{0}^{B}|F(u ; b)| d u\right) \\
\ll & Q^{-1}\left(|F(B ; a)||F(B ; b)|+Q^{-1} \int_{0}^{B}(|F(u ; a)||F(B ; b)|\right. \\
& \left.+|F(B ; a)||F(u ; b)|) d u+Q^{-2} \int_{0}^{B} \int_{0}^{B}|F(u ; a)||F(v ; b)| d u d v\right) .
\end{aligned}
$$

Воспользовавшись тем, что при любых $u$ и $v$

$$
|F(u ; a)||F(v ; b)| \leqslant|F(u ; a)|^{2}+|F(v ; b)|^{2}
$$


преобразуем неравенство для $V_{2}(a, b)$ к виду

$$
\left|V_{2}(a, b)\right| \ll Q^{-1}\left(|F(B ; a)|^{2}+|F(B ; b)|^{2}+Q^{-1} \int_{0}^{B}\left(|F(u ; a)|^{2}+|F(u ; b)|^{2}\right) d u\right) .
$$

Собирая полученные оценки вместе и пользуясь тем, что $Q^{-1} \ll \sqrt{x} X^{-0.5}$, заключаем, что

$$
\varrho(\sigma, T) \ll X^{-0.5} \sqrt{x} K(T)
$$

где

$$
\begin{aligned}
K(T)= & \sum_{\gamma} \sum_{a, b=-B}^{B}(|a|+1)^{-1}(|b|+1)^{-1} \int_{-2 H}^{2 H} \int_{-H_{1}}^{H_{1}} \ldots \int_{-H_{1}}^{H_{1}}\left(|F(B ; a)|^{2}\right. \\
& \left.+|F(B ; b)|^{2}+Q^{-1} \int_{0}^{B}\left(|F(u ; a)|^{2}+|F(u ; b)|^{2}\right) d u\right) d t d t_{1} \ldots d t_{k} .
\end{aligned}
$$

Положим

$$
I(u ; c)=\int_{X}^{2 X}|F(u ; c)|^{2} d T .
$$

Согласно лемме $4 I(u ; c) \ll X \sqrt{X} x^{3} H^{-1}$. Используя эту оценку, находим

$$
\begin{aligned}
\int_{X}^{2 X} K(T) d T \ll & \sum_{\gamma} \sum_{a, b=-B}^{B}(|a|+1)^{-1}(|b|+1)^{-1} \\
& \times \int_{-2 H}^{2 H} \int_{-H_{1}}^{H_{1}} \ldots \int_{-H_{1}}^{H_{1}} X \sqrt{X} x^{3} H^{-1}\left(1+Q^{-1} B\right) d t d t_{1} \ldots d t_{k} \\
\ll & H D X \sqrt{X} x^{3} H^{-1}(x L)^{2} \ll H D X \sqrt{X} x^{6} H^{-1} .
\end{aligned}
$$

Пусть $G$ - множество тех $T, X \leqslant T \leqslant 2 X$, для которых

$$
K(T)>H D \sqrt{X} x^{6} H^{-0.9} .
$$

Чтобы оценить меру множества $G$, проинтегрируем по $G$ последнее неравенство и воспользуемся полученной вьше оценкой:

$$
H D \sqrt{X} x^{6} H^{-0.9} \mu(G) \leqslant \int_{G} K(T) d T \leqslant \int_{X}^{2 X} K(T) d T \ll H D X \sqrt{X} x^{6} H^{-1},
$$

откуда

$$
H D \sqrt{X} x^{6} H^{-0.9} \mu(G) \ll H D X \sqrt{X} x^{6} H^{-1} .
$$

Производя сокрашения, находим $\mu(G) \ll X H^{-0.1}$. Для завершения доказательства леммы остается лишь заметить, что для $T$, не принадлежаших множеству $G$, справедливы оценки

$$
K(T) \leqslant H D \sqrt{X} x^{6} H^{-0.9}
$$

И

$$
\varrho(\sigma, T) \ll H D x^{6} \sqrt{x} H^{-0.9} \ll H D H^{-0.8},
$$

где $\sigma$ - любое число, удовлетворяюшее условию $0.5 \leqslant \sigma \leqslant 1$.

ЗАмечание. Нетривиальные оценки сумм, подобных $r\left(\sigma_{1}, T\right)$ и $\varrho(\sigma, T)$, были получены А. А. Карацубой [5] для случая $H \geqslant T^{27 / 82+\alpha}, 0<\alpha<0.001$. Утверждения лемм этого параграфа отражают тот факт, что для "почти всех" $T$ суммы $r\left(\sigma_{1}, T\right)$ и $\varrho(\sigma, T)$ при любых рассматриваемых $\sigma_{1}$ и $\sigma$ допускают нетривиальную оценку уже для $H \geqslant T^{\varepsilon}$. 


\section{§ 4. Доказательство теоремы А}

Доказательству основного утверждения этого параграфа предпошлем несколько вспомогательных лемм.

Лемма 7. Пусть $s=\sigma+i t, s \neq 1, y \geqslant 1$. Тогда равномерно по $\sigma \geqslant 0$, $|t| \leqslant y$

$$
\zeta(s)=\sum_{n \leqslant y} n^{-s}-\frac{y^{1-s}}{1-s}+O\left(y^{-\sigma}\right)
$$

Лемма 8. Пусть $s=\sigma+i t, 0.5 \leqslant \sigma \leqslant 1, y \geqslant 1, z \geqslant 1 u t=2 \pi y z$. Тогда справедливо следующее равенство:

$$
\zeta(s)=\sum_{n \leqslant y} n^{-s}+\chi(s) \sum_{n \leqslant z} n^{s-1}+O\left(y^{-\sigma}\right)+O\left(z^{\sigma-1} t^{0.5-\sigma}\right),
$$

$2 \partial e$

$$
\chi(s)=e^{\frac{\pi i}{4}}\left(\frac{t}{2 \pi}\right)^{0.5-\sigma}\left(\frac{t}{2 \pi e}\right)^{-i t},
$$

а постоянные в знаках $O-$ абсолютные.

ДокАЗАТЕЛЬСтВА этих утверждений см. в [3], [11], [12].

ЛЕмма 9. Пусть $0.5 \leqslant \sigma \leqslant 1, \quad|t-T| \leqslant 3 H, \gamma-$ рачиональная дробь, числитель и знаменатель которой - натуральнье числа, не превосходящие $x$, $P_{1}=P \sqrt{\gamma}, P_{2}=P / \sqrt{\gamma}$. Тогда имеет место равенство

$$
\zeta(\sigma+i t)=\sum_{n \leqslant P_{1}} n^{-\sigma-i t}+\vartheta P^{1-2 \sigma-2 i t} \sum_{n \leqslant P_{2}} n^{\sigma-1+i t}+O\left(X^{-0.2}\right)
$$

əде $\vartheta=\exp (i(\pi / 4+T))$.

ДоКАЗАТЕЛЬСТво. Определим величину $x_{1}$ из соотношения $2 \pi P x_{1}=t$ и положим в предыдущей лемме $y=x_{1} \sqrt{\gamma}, z=P_{2}$. Таким образом получим равенство

$$
\zeta(\sigma+i t)=\sum_{n \leqslant x_{1} \sqrt{\gamma}} n^{-\sigma-i t}+\chi(\sigma+i t) \sum_{n \leqslant P_{2}} n^{\sigma-1+i t}+R
$$

в котором

$$
R \ll\left(x_{1} \sqrt{\gamma}\right)^{-\sigma}+P_{2}^{\sigma-1} T^{0.5-\sigma} \ll T^{-0.5 \sigma}\left(\gamma^{-\sigma / 2}+\gamma^{(1-\sigma) / 2}\right) \ll X^{-0.2}
$$

Поскольку

$$
\left|x_{1} \sqrt{\gamma}-P_{1}\right|=\sqrt{\gamma}\left|x_{1}-P\right|=\sqrt{\gamma}\left|\frac{t}{2 \pi P}-P\right|=\frac{\sqrt{\gamma}}{2 \pi P}|t-T| \leqslant \frac{3 H \sqrt{\gamma}}{2 \pi P}<1
$$


то при замене $x_{1} \sqrt{\gamma}$ на $P_{1}$ в формуле для $\zeta(\sigma+i t)$ соответствуюшая сумма может измениться лишь на одно последнее слагаемое, величина которого есть $O\left(\left(x_{1} \sqrt{\gamma}\right)^{-\sigma}\right)=O\left(X^{-0.2}\right)$. Поэтому выражение для $\zeta(\sigma+i t)$ принимает вид

$$
\zeta(\sigma+i t)=\sum_{n \leqslant P_{1}} n^{-\sigma-i t}+\chi(\sigma+i t) \sum_{n \leqslant P_{2}} n^{\sigma-1+i t}+O\left(X^{-0.2}\right) .
$$

Для завершения доказательства остается показать, что множитель $\chi(\sigma+i t)$ в этом выражении может быть заменен на величину $\vartheta P^{1-2 \sigma-2 i t}$. Прежде всего, из теоремы Лагранжа о конечных приращениях следует, что

$$
\left(\frac{t}{2 \pi}\right)^{0.5-\sigma}-\left(\frac{T}{2 \pi}\right)^{0.5-\sigma}=O\left(H T^{-0.5-\sigma}\right)=O\left(H X^{-1}\right) .
$$

Поэтому

$$
\chi(\sigma+i t)=e^{\pi i / 4} P^{1-2 \sigma}\left(\frac{t}{2 \pi e}\right)^{-i t}+O\left(H X^{-1}\right) .
$$

Далее, для рассматриваемых значений $T$ имеем

$$
\begin{aligned}
t^{i t} & =T^{i t}\left(\frac{t}{T}\right)^{i t}=T^{i t} \exp \left(i t \ln \left(1+\frac{t-T}{T}\right)\right) \\
& =T^{i t} \exp \left(i t\left(\frac{t-T}{T}+O\left(H^{2} X^{-2}\right)\right)\right)=T^{i t} \exp \left(\frac{i t(t-T)}{T}\right)+O\left(H^{2} X^{-1}\right) \\
& =T^{i t} \exp \left(\frac{i(T+(t-T))(t-T)}{T}\right)+O\left(H^{2} X^{-1}\right) \\
& =T^{i t} e^{i(t-T)} \exp \left(\frac{i(t-T)^{2}}{T}\right)+O\left(H^{2} X^{-1}\right)=e^{-i T}(e T)^{i t}+O\left(H^{2} X^{-1}\right)
\end{aligned}
$$

Поэтому

$$
\left(\frac{t}{2 \pi e}\right)^{-i t}=e^{i T} P^{-2 i t}+O\left(H^{2} X^{-1}\right)
$$

и

$$
\chi(\sigma+i t)=e^{i(\pi / 4+T)} P^{1-2 \sigma-2 i t}+O\left(H^{2} X^{-1}\right) .
$$

Подставляя это выражение в формулу для $\zeta(\sigma+i t)$ и пользуясь тем, что

$$
H^{2} X^{-1} \sum_{n \leqslant P_{2}} n^{\sigma-1} \ll H^{2} X^{-1} X^{0.5 \sigma} \sqrt{x} \ll X^{-0.2},
$$

приходим к утверждению леммы.

Следуя работе [1], рассмотрим “успокаивающую” функцию $\eta_{\sigma}(s)=\eta(s)$. Пусть $0<\alpha \leqslant 1$ и

$$
\varphi_{\alpha}(n)=n^{\alpha} \prod_{p \mid n}\left(1-p^{-\alpha}\right) .
$$


Для $0.5<\sigma \leqslant 1$ определим числа $\lambda_{\nu}, \nu=1,2, \ldots,[x]$, равенствами

$$
\begin{aligned}
\lambda_{\nu} & =\nu^{2 \sigma}\left(\sum_{d<x} \frac{\mu^{2}(d)}{\varphi_{2 \sigma}(d)}\right)^{-1} \sum_{d<x \nu^{-1}} \frac{\mu(d \nu) \mu(d)}{\varphi_{2 \sigma}(d)} \\
& =\frac{\mu(\nu) \nu^{2 \sigma}}{\varphi_{2 \sigma}(\nu)}\left(\sum_{d<x} \frac{\mu^{2}(d)}{\varphi_{2 \sigma}(d)}\right)^{-1} \sum_{\substack{d<x \nu^{-1} \\
(d, \nu)=1}} \frac{\mu^{2}(d)}{\varphi_{2 \sigma}(d)} .
\end{aligned}
$$

Тогда $\lambda_{1}=1$ и для $\nu>1$

$$
\left|\lambda_{\nu}\right| \leqslant \frac{\nu^{2 \sigma}}{\varphi_{2 \sigma}(\nu)}=\prod_{p \mid \nu}\left(1-p^{-2 \sigma}\right)^{-1} \ll \prod_{p<x}\left(1+p^{-1}\right) \ll \ln x \ll L .
$$

Функиией Сельберга назовем функцию

$$
\eta_{\sigma}(s)=\eta(s)=\sum_{\nu<x} \lambda_{\nu} \nu^{-s}
$$

ЛЕмма 10. Справедливы соотношения

1) $\sum_{\nu_{1}, \nu_{2}<x} \frac{\lambda_{\nu_{1}} \lambda_{\nu_{2}}}{\left(\nu_{1} \nu_{2}\right)^{2 \sigma}}\left(\nu_{1}, \nu_{2}\right)^{2 \sigma}=\left(\sum_{d<x} \frac{\mu^{2}(d)}{\varphi_{2 \sigma}(d)}\right)^{-1}$;

2) $\zeta(2 \sigma)\left(\sum_{d<x} \frac{\mu^{2}(d)}{\varphi_{2 \sigma}(d)}\right)^{-1}<1+O\left(x^{1-2 \sigma}\right)$;

3) $\sum_{\nu_{1}, \nu_{2}<x} \frac{\lambda_{\nu_{1}} \lambda_{\nu_{2}}}{\nu_{1} \nu_{2}}\left(\nu_{1}, \nu_{2}\right)^{2-2 \sigma}>0$

Доказательства этих утверждений см. в [1].

ЛЕмма 11. Пусть $\Phi(s)=\zeta(s) \eta(s)$, u nycmb

$$
I(\sigma, T)=D^{-1} \int_{-2 H}^{2 H} \int_{-H_{1}}^{H_{1}} \ldots \int_{-H_{1}}^{H_{1}}\left(\left|\Phi\left(\sigma+i T_{1}\right)\right|^{2}-1\right) d t d t_{1} \ldots d t_{k}
$$

$T_{1}=T+t+t_{1}+\cdots+t_{k}$. Тогда для всех $T$ из промежутка $(X, 2 X]$, за исключением значений, образующих множество $\mathscr{E}$ с мерой $\mu(\mathscr{E}) \ll X H^{-0.1}$, при любом $\sigma, 0.5 \leqslant \sigma \leqslant 1$, имеет место неравенство

$$
I(\sigma, T) \ll H x^{-(2 \sigma-1)} .
$$


ДокАЗАТЕЛЬСтво. Воспользовавшись определением функции Сельберга $\eta_{\sigma}(s)$, представим исходный интеграл в виде суммы:

$I=D^{-1} \sum_{\nu_{1}, \nu_{2}<x}\left(\nu_{1} \nu_{2}\right)^{-\sigma} \lambda_{\nu_{1}} \lambda_{\nu_{2}} \int_{-2 H}^{2 H} \int_{-H_{1}}^{H_{1}} \ldots \int_{-H_{1}}^{H_{1}}\left|\zeta\left(\sigma+i T_{1}\right)\right|^{2} \beta^{i T_{1}} d t d t_{1} \ldots d t_{k}-1$

где $\beta=\nu_{1} \nu_{2}^{-1}=\mu_{1} \mu_{2}^{-1}$, a $\mu_{r}=\nu_{r}\left(\nu_{1}, \nu_{2}\right)^{-1}, r=1,2$. Полагая в лемме $8 t=T_{1}$, $P_{1}=P \sqrt{\beta}, P_{2}=P / \sqrt{\beta}, \gamma=\beta, \gamma=\beta^{-1}$ и замечая, что

$$
\left|T_{1}-T\right|=\left|t+t_{1}+\cdots+t_{k}\right| \leqslant 2 H+k H_{1} \leqslant 3 H
$$

приходим к равенствам

$$
\zeta\left(\sigma+i T_{1}\right)=\sum_{n \leqslant P_{1}} n^{-\sigma-i T_{1}}+\vartheta P^{1-2 \sigma-2 i T_{1}} \sum_{n \leqslant P_{2}} n^{\sigma-1+i T_{1}}+O\left(X^{-0.2}\right)
$$

И

$$
\zeta\left(\sigma-i T_{1}\right)=\sum_{m \leqslant P_{1}} m^{-\sigma+i T_{1}}+\bar{\vartheta} P^{1-2 \sigma+2 i T_{1}} \sum_{m \leqslant P_{2}} m^{\sigma-1-i T_{1}}+O\left(X^{-0.2}\right),
$$

в которых $\vartheta=e^{i(\pi / 4+T)}$. Перемножим их почленно и воспользуемся оценкой $\left|\zeta\left(\sigma+i T_{1}\right)\right| \ll X^{1 / 6}$, справедливой при $\sigma \geqslant 0.5$ и $T_{1} \asymp X$ (см. [3, с. 117-119]). Таким образом получим

$$
\left|\zeta\left(\sigma+i T_{1}\right)\right|^{2} \beta^{i T_{1}}=S_{1}+P^{2(1-2 \sigma)} S_{2}+P^{1-2 \sigma}\left(\bar{\vartheta} \bar{\Sigma}_{1}+\vartheta \Sigma_{2}\right)+O\left(X^{-1 / 30}\right),
$$

где

$$
\begin{aligned}
& S_{1}=\sum_{n \leqslant P_{1}} \sum_{m \leqslant P_{2}}(m n)^{-\sigma}\left(\frac{m \beta}{n}\right)^{i T_{1}}, \\
& S_{2}=\sum_{m \leqslant P_{1}} \sum_{n \leqslant P_{2}}(m n)^{\sigma-1}\left(\frac{n \beta}{m}\right)^{i T_{1}},
\end{aligned}
$$

a

$$
\Sigma_{\nu}=\sum_{m, n \leqslant P_{\nu}} m^{\sigma-1} n^{-\sigma}\left(\frac{m n}{P_{\nu}^{2}}\right)^{i T_{1}}, \quad \nu=1,2
$$

Область изменения $m$ и $n$ в сумме $S_{1}$ разобьем на пять частей: $m \beta=n, m \beta<$ $n \leqslant m \beta(1+\Delta), n<m \beta \leqslant n(1+\Delta), m \beta(1+\Delta)<n$ и $m \beta>n(1+\Delta)$. Используя обозначения леммы 5 , представим $S_{1}$ в виде

$$
S_{1}=A_{1}+\beta^{i T_{1}}\left(A_{2}+A_{3}\right)+A_{4}+A_{5}
$$


где

$$
\begin{gathered}
A_{1}=\sum_{\substack{n \leqslant P_{1}, m \leqslant P_{2} \\
m \beta=n}}(m n)^{-\sigma}, \\
A_{2}=\sum_{m \leqslant P_{2}} \sum_{n \leqslant P_{1}}(m n)^{-\sigma} \Phi(m, n ; \beta)\left(\frac{m}{n}\right)^{i T_{1}}=W\left(\sigma, T_{1} ; \beta\right), \\
A_{3}=\sum_{n \leqslant P_{1}} \sum_{m \leqslant P_{2}}(m n)^{-\sigma} \Phi\left(n, m ; \beta^{-1}\right)\left(\frac{m}{n}\right)^{i T_{1}}=\bar{W}\left(\sigma, T_{1} ; \beta^{-1}\right), \\
A_{4}=\sum_{m \leqslant P_{2}} m \beta(1+\Delta)<n \leqslant P_{1} \\
A_{5}=\sum_{n \leqslant P_{1}}(m n)^{-\sigma}\left(\frac{m \beta}{n}\right)^{i T_{1}}, \\
\sum_{n \rightarrow \Delta)<m \leqslant P_{2}}(m n)^{-\sigma}\left(\frac{m \beta}{n}\right)^{i T_{1}} .
\end{gathered}
$$

Подобным образом представляем сумму

$$
S_{2}=B_{1}+\beta^{i T_{1}}\left(B_{2}+B_{3}\right)+B_{4}+B_{5},
$$

где

$$
\begin{gathered}
B_{1}=\sum_{\substack{m \leqslant P_{1}, n \leqslant P_{2} \\
n \beta=m}}(m n)^{\sigma-1}, \\
B_{2}=W\left(1-\sigma, T_{1} ; \beta\right), \quad B_{3}=\bar{W}\left(1-\sigma, T_{1} ; \beta^{-1}\right), \\
B_{4}=\sum_{n \leqslant P_{2}} \sum_{n \beta(1+\Delta)<m \leqslant P_{1}}(m n)^{\sigma-1}\left(\frac{n \beta}{m}\right)^{i T_{1}}, \\
B_{5}=\sum_{m \leqslant P_{1}} \sum_{m \beta^{-1}(1+\Delta)<n \leqslant P_{2}}(m n)^{\sigma-1}\left(\frac{n \beta}{m}\right)^{i T_{1}} .
\end{gathered}
$$

Наконец, каждую из сумм $\Sigma_{\nu}, \nu=1,2$, преобразуем с использованием леммы 6 :

$$
\Sigma_{\nu}=C_{\nu}+P_{\nu}^{-2 i T_{1}} D_{\nu}
$$

где

$$
\begin{aligned}
C_{\nu} & =\sum_{\min (m, n) \leqslant P_{\nu}(1-\Delta)} m^{\sigma-1} n^{-\sigma}\left(\frac{m n}{P_{\nu}^{2}}\right)^{i T_{1}}, \\
D_{\nu} & =\sum_{P_{\nu}(1-\Delta)<m, n \leqslant P_{\nu}} m^{\sigma-1} n^{-\sigma}(m n)^{i T_{1}} .
\end{aligned}
$$


Суммы $A_{1}$ и $B_{1}$ вычислим, используя лемму 7 . Из определения $\mu_{1}$ и $\mu_{2}$ следует, что $\left(\mu_{1}, \mu_{2}\right)=1$. Поэтому, переписывая равенство $m \beta=n$ в виде $m \mu_{1}=n \mu_{2}$, заключаем, что $m=\nu \mu_{2}$ и $n=\nu \mu_{1}$, где $\nu$ - целое число, удовлетворяющее условию $1 \leqslant \nu \leqslant P_{0}, P_{0}=P / \sqrt{\mu_{1} \mu_{2}}$. Таким образом,

$$
\begin{aligned}
A_{1} & =\sum_{1 \leqslant \nu \leqslant P_{0}}\left(\mu_{1} \mu_{2} \nu^{2}\right)^{-\sigma}=\left(\mu_{1} \mu_{2}\right)^{-\sigma} \sum_{\nu \leqslant P_{0}} \nu^{-2 \sigma} \\
& =\left(\mu_{1} \mu_{2}\right)^{-\sigma} \zeta(2 \sigma)+\frac{1}{\sqrt{\mu_{1} \mu_{2}}} \frac{P^{1-2 \sigma}}{1-2 \sigma}+O\left(X^{-\sigma}\right) .
\end{aligned}
$$

Поскольку $B_{1}$ можно получить из $A_{1}$, меняя $\sigma$ на $1-\sigma$, и поскольку $1-\sigma \geqslant 0$, Tо

$$
B_{1}=\left(\mu_{1} \mu_{2}\right)^{\sigma-1} \zeta(2-2 \sigma)+\frac{1}{\sqrt{\mu_{1} \mu_{2}}} \frac{P^{2 \sigma-1}}{2 \sigma-1}+O\left(X^{\sigma-1}\right) .
$$

Подставляя в полученные равенства вместо $\mu_{1}$ и $\mu_{2}$ их выражения через $\nu_{1}$ и $\nu_{2}$, находим

$$
A_{1}+P^{2(1-2 \sigma)} B_{1}=\zeta(2 \sigma) \frac{\left(\nu_{1}, \nu_{2}\right)^{\sigma}}{\left(\nu_{1} \nu_{2}\right)^{2 \sigma}}+P^{2(1-2 \sigma)} \zeta(2-2 \sigma) \frac{\left(\nu_{1}, \nu_{2}\right)^{2-2 \sigma}}{\left(\nu_{1} \nu_{2}\right)^{1-\sigma}}+O\left(X^{-\sigma}\right) .
$$

Вклад этих слагаемых в исходный интеграл $I$ равен

$$
\begin{aligned}
I_{0}= & 4 H\left(\zeta(2 \sigma) \sum_{\nu_{1}, \nu_{2}<x} \frac{\lambda_{\nu_{1}} \lambda_{\nu_{2}}}{\left(\nu_{1} \nu_{2}\right)^{2 \sigma}}\left(\nu_{1}, \nu_{2}\right)^{\sigma}+P^{2(1-2 \sigma)} \zeta(2-2 \sigma)\right. \\
& \left.\times \sum_{\nu_{1}, \nu_{2}<x} \frac{\lambda_{\nu_{1}} \lambda_{\nu_{2}}}{\nu_{1} \nu_{2}}\left(\nu_{1}, \nu_{2}\right)^{2-2 \sigma}+O\left(\sum_{\nu_{1}, \nu_{2}<x} \frac{\left|\lambda_{\nu_{1}} \lambda_{\nu_{2}}\right|}{\sqrt{\nu_{1} \nu_{2}}}\right)\right) .
\end{aligned}
$$

Согласно утверждениям 1) и 2) леммы 10 для $0.5<\sigma \leqslant 1$ имеем

$$
\zeta(2 \sigma) \sum_{\nu_{1}, \nu_{2}<x} \frac{\lambda_{\nu_{1}} \lambda_{\nu_{2}}}{\left(\nu_{1} \nu_{2}\right)^{2 \sigma}}\left(\nu_{1}, \nu_{2}\right)^{\sigma}=\zeta(2 \sigma)\left(\sum_{\nu<x} \frac{\mu^{2}(\nu)}{\varphi_{2 \sigma}(\nu)}\right)^{-1}<1+O\left(x^{1-2 \sigma}\right) .
$$

Кроме того, в силу утверждения 3 ) той же леммы следует, что второе слагаемое в выражении для $I_{0}$ отрицательно, так как $\zeta(2-2 \sigma)<0$ при $0.5<\sigma \leqslant 1$. Действительно, при $\operatorname{Re} s>0$

$$
\zeta(s)=\frac{1}{2}+\frac{1}{s-1}+s \int_{1}^{+\infty} \rho(u) u^{-s-1} d u, \quad \rho(u)=\frac{1}{2}-\{u\}
$$

(см. [13, с. 33]), так что для $0<\sigma_{1}<1$

$$
\zeta\left(\sigma_{1}\right)=\frac{1}{2}+\frac{1}{\sigma_{1}-1}+\sigma_{1} \int_{1}^{+\infty} \rho(u) u^{-\sigma_{1}-1} d u .
$$

Поскольку $|\rho(u)| \leqslant 0.5$, то

$$
\left|\sigma_{1} \int_{1}^{+\infty} \rho(u) u^{-\sigma_{1}-1} d u\right| \leqslant 0.5 \sigma_{1} \int_{1}^{+\infty} u^{-\sigma_{1}-1} d u=0.5
$$




$$
\zeta\left(\sigma_{1}\right) \leqslant \frac{1}{2}-\frac{1}{1-\sigma_{1}}+\frac{1}{2}=-\frac{\sigma_{1}}{1-\sigma_{1}}<0 .
$$

Поэтому

$$
I_{0}<4 H\left(1+O\left(x^{1-2 \sigma}\right)+O\left(X^{-\sigma} x L^{2}\right)\right)=4 H\left(1+O\left(x^{1-2 \sigma}\right)\right)
$$

Обозначим через $I_{1}$ вклад в $I(\sigma, T)$ от сумм $A_{2}, A_{3}, B_{2}, B_{3}, D_{1}$ и $D_{2}$, а через $I_{2}$ - вклад от сумм $A_{4}, A_{5}, B_{4}, B_{5}, C_{1}$ и $C_{2}$. Оценим сначала $I_{1}$. Поскольку $\left|\lambda_{\nu_{1}} \lambda_{\nu_{2}}\left(\nu_{1} \nu_{2}\right)^{-\sigma}\right| \ll L^{2}$ при $0.5 \leqslant \sigma \leqslant 1$ и при изменении $\nu_{1}$ и $\nu_{2}$ в пределах от 1 до $x$ величины $\beta=\nu_{1} \nu_{2}^{-1}$ и $\beta^{-1}=\nu_{2} \nu_{1}^{-1}$ пробегают одно и то же множество значений, то приходим к неравенству

$$
\left|I_{1}\right| \ll D^{-1} L^{2}\left(r(\sigma, T)+P^{1-2 \sigma} r(1-\sigma, T)+\varrho(\sigma, T)\right),
$$

где

$$
\begin{aligned}
r\left(\sigma_{1}, T\right) & =\sum_{\nu_{1}, \nu_{2}<x} \int_{-2 H}^{2 H} \int_{-H_{1}}^{H_{1}} \ldots \int_{-H_{1}}^{H_{1}}\left|W\left(\sigma_{1}, T_{1} ; \beta\right)\right| d t d t_{1} \ldots d t_{k}, \\
\varrho(\sigma, T) & =\sum_{\nu_{1}, \nu_{2}<x} \int_{-2 H}^{2 H} \int_{-H_{1}}^{H_{1}} \ldots \int_{-H_{1}}^{H_{1}}\left|V\left(\sigma, T_{1} ; \beta\right)\right| d t d t_{1} \ldots d t_{k} .
\end{aligned}
$$

Согласно утверждению леммы 5 найдутся такие множества $E_{1}$ и $E_{2}$, что мера каждого из них по порядку не превосходит величины $X H^{-0.1}$ и при любом $\sigma, 0.5 \leqslant$ $\sigma \leqslant 1$, справедливы соотношения

$$
\begin{aligned}
r(\sigma, T) & \ll H D H^{-0.3}, & \text { если } T \notin E_{1}, \\
r(1-\sigma, T) & \ll H D X^{\sigma-0.5} H^{-0.3}, & \text { если } T \notin E_{2} .
\end{aligned}
$$

Кроме того, по лемме 6 существует множество $G$ такое, что $\mu(G) \ll X H^{-0.1}$ и на дополнении $G$ до всего промежутка $(X, 2 X]$ при любом рассматриваемом $\sigma$ будет выполнено неравенство

$$
\varrho(\sigma, T) \ll H D H^{-0.8}
$$

Обозначая буквой $\mathscr{E}$ объединение множеств $E_{1}, E_{2}$ и $G$, заключаем, что для всех $T, X \leqslant T \leqslant 2 X$, кроме значений из множества $\mathscr{E}$, при любом $\sigma$, удовлетворяющем условию $0.5 \leqslant \sigma \leqslant 1$, будет верна оценка

$$
\begin{aligned}
I_{1} & \ll D^{-1} L^{2}\left(H D H^{-0.3}+P^{1-2 \sigma} H D X^{\sigma-0.5} H^{-0.3}+H D H^{-0.8}\right) \\
& \ll H L^{2} H^{-0.3} \ll H H^{-0.2} .
\end{aligned}
$$

Оценим теперь величину $I_{2}$. Пусть числа $m$ и $n$ отвечают какому-либо слагаемому из $A_{4}$, так что $n>m \beta(1+\Delta)$. Тогда для этих $m$ и $n$

$$
\left|\ln \frac{m \beta}{n}\right|>\ln (1+\Delta)>0.5 \Delta .
$$


Значит, при $H_{2}=2 H, H_{1}$ вьполняются неравенства

$$
\begin{aligned}
\left|\int_{-H_{2}}^{H_{2}}\left(\frac{m \beta}{n}\right)^{i u} d u\right| & =\left|\left(\frac{m \beta}{n}\right)^{i H_{2}}-\left(\frac{m \beta}{n}\right)^{-i H_{2}}\right|\left|\ln \frac{m \beta}{n}\right|^{-1} \\
& <2(0.5 \Delta)^{-1}=4 \Delta^{-1}=4 H_{1} L^{-1}
\end{aligned}
$$

Интегрируя по $t, t_{1}, \ldots, t_{k}$ каждое из таких слагаемых, убеждаемся, что вклад от них в исходный интеграл не превосходит величины

$$
\begin{aligned}
D^{-1} \sum_{\nu_{1}, \nu_{2}<x} \frac{\left|\lambda_{\nu_{1}} \lambda_{\nu_{2}}\right|}{\left(\nu_{1} \nu_{2}\right)^{\sigma}}\left(4 H_{1} L^{-1}\right)^{k+1} \sum_{n \leqslant P_{1}} \sum_{m \leqslant P_{2}}(m n)^{-\sigma} \\
\ll D^{-1} D H(0.5 L)^{-k-2} x L^{2}\left(P_{1} P_{2}\right)^{1-\sigma} \ll H X^{-1} .
\end{aligned}
$$

Сходными рассуждениями можно показать, что вклад от сумм $A_{5}, B_{4}$ и $B_{5}$ также оценивается сверху величиной $H X^{-1}$.

Пусть теперь $\nu$ равно 1 или 2 , а $m$ и $n$ таковы, что $\min (m, n) \leqslant P_{\nu}(1-\Delta)$. Тогда $m n \leqslant P_{\nu}^{2}(1-\Delta)$ и

$$
\left|\ln \frac{m n}{P_{\nu}^{2}}\right| \geqslant-\ln (1-\Delta)>\Delta .
$$

Значит, при $H_{2}=2 H, H_{1}$ справедливы неравенства

$$
\left|\int_{-H_{2}}^{H_{2}}\left(\frac{m n}{P_{\nu}^{2}}\right)^{i u} d u\right|=\left|\left(\frac{m n}{P_{\nu}^{2}}\right)^{i H_{2}}-\left(\frac{m n}{P_{\nu}^{2}}\right)^{-i H_{2}}\right|\left|\ln \frac{m n}{P_{\nu}^{2}}\right|^{-1}<2 \Delta^{-1}=2 H_{1} L^{-1} .
$$

Проинтегрировав каждое из слагаемых $D_{1}$ и $D_{2}$ по $t, t_{1}, \ldots, t_{k}$, заключаем, что вклад от $D_{1}$ и $D_{2}$ не превосходит величины

$$
D^{-1} P^{1-2 \sigma} \sum_{\nu_{1}, \nu_{2}<x} \frac{\left|\lambda_{\nu_{1}} \lambda_{\nu_{2}}\right|}{\left(\nu_{1} \nu_{2}\right)^{\sigma}} \sum_{m, n \leqslant P_{1}+P_{2}} m^{\sigma-1} n^{-\sigma}\left(2 H_{1} L^{-1}\right)^{k+1} \ll H X^{-1} .
$$

Собирая полученные оценки вместе и предполагая, что $T$ принадлежит множеству $\mathscr{E} 1$ - дополнению множества $\mathscr{E}$ до промежутка $(X, 2 X]$, приходим к неравенству, справедливому для любого $\sigma$, удовлетворяюшего условию $0.5<\sigma \leqslant 1$ :

$$
I(\sigma, T)<4 H\left(1+O\left(x^{1-2 \sigma}\right)\right)+O\left(H H^{-0.2}\right)+O\left(H X^{-1}\right)-4 H=O\left(H x^{1-2 \sigma}\right) .
$$

Лемма доказана.

ЛЕмма 12. Пусть

$$
I=\int_{\sigma}^{1}(N(u, T+H)-N(u, T-H)) d u .
$$

Тогда для всех T, принадлежащих множеству Ё из предыдущей леммы, при любом $\sigma$, удовлетворяющем условию $0.5 \leqslant \sigma \leqslant 1$, верна оценка

$$
I \ll H x^{1-2 \sigma} .
$$


ДокАЗАТЕЛЬСтво. Пусть $T$ принадлежит множеству $\mathscr{E}_{1}$. Рассмотрим сначала $\sigma$ с условием $0.5+L^{-1} \leqslant \sigma \leqslant 1$. Воспользуемся неравенством, вывод которого содержится в работе [5]:

$$
I \leqslant(2 \pi D)^{-1} \int_{-2 H}^{2 H} \int_{-H_{1}}^{H_{1}} \cdots \int_{-H_{1}}^{H_{1}}\left(\left|\Phi\left(\sigma+i T_{1}\right)\right|^{2}-1\right) d t d t_{1} \ldots d t_{k}+O(L),
$$

где $\Phi(s)=\zeta(s) \eta_{\sigma}(s), T_{1}=T+t+t_{1}+\cdots+t_{k}$. Применяя к последнему интегралу лемму 11, придем к искомому неравенству

$$
I \ll H x^{1-2 \sigma}+L \ll H x^{1-2 \sigma} .
$$

Пусть теперь $0.5 \leqslant \sigma \leqslant 0.5+L^{-1}$. Тогда интеграл $I$ из условия леммы можно представить в виде

$$
I=I_{1}+I_{2}
$$

где

$$
\begin{aligned}
& I_{1}=\int_{\sigma}^{0.5+L^{-1}}(N(u, T+H)-N(u, T-H)) d u, \\
& I_{2}=\int_{0.5+L^{-1}}^{1}(N(u, T+H)-N(u, T-H)) d u .
\end{aligned}
$$

Первый интеграл оценивается тривиально:

$$
I_{1} \leqslant \int_{0.5}^{0.5+L^{-1}}(N(T+H)-N(T-H)) d u \ll L^{-1} H L \ll H .
$$

Кроме того, по доказанному вьше

$$
I_{2} \ll H x^{1-2\left(0.5+L^{-1}\right)} \ll H .
$$

Таким образом, $I \ll H+H \ll H$ и исходное неравенство доказано для всех $\sigma$ из промежутка $0.5 \leqslant \sigma \leqslant 1$.

ДоКАЗАТЕЛЬСТВо тЕОРЕМЫ А. Покажем, что определенное вьше множество $\mathscr{E}_{1}$ обладает свойствами, указанными в условии теоремы. Пусть $T$ принадлежит $\mathscr{E}_{1}$. Если $0.5 \leqslant \sigma \leqslant 0.5+L^{-1}$, то соответствуюшая оценка является следствием формулы Римана-Мангольдта для $N(T)$; см. [3, с. 211]. Поэтому нам достаточно рассмотреть случай $0.5+L^{-1}<\sigma \leqslant 1$. Поскольку функция $f(u)=N(u, T+H)-$ $N(u, T-H)$ не возрастает с ростом $u$, то справедливы следующие неравенства:

$$
N(\sigma, T+H)-N(\sigma, T-H) \leqslant L \int_{\sigma-L^{-1}}^{\sigma} f(u) d u \leqslant L \int_{\sigma-L^{-1}}^{1} f(u) d u .
$$

Так как $\sigma-L^{-1} \geqslant 0.5$, то для оценки последнего интеграла можно применить лемму 12:

$N(\sigma, T+H)-N(\sigma, T-H) \ll L H x^{1-2\left(\sigma-L^{-1}\right)} \ll H x^{1-2 \sigma} L \ll H X^{-0.01 \varepsilon(2 \sigma-1)} L$.

Для завершения доказательства осталось заметить, что

$$
\mu\left(\mathscr{E}_{1}\right)=X-O\left(X H^{-0.1}\right) .
$$

Теорема доказана. 


\section{$\S 5$. Моменты величин $S(t)$ и $S_{1}(t+h)-S_{1}(t)$}

Этот параграф̆ посвящен вычислению моментов функций $S(t)$ и $S_{1}(t+h)-S_{1}(t)$ на коротких промежутках вида $(T, T+H]$ в случае, когда $T$ принадлежит множеству $\mathscr{E}_{1}$, введенному в теореме А. Основные утверждения этого параграфа - теоремы 1 и 2 - будут использованы для нижней оценки разности $M(T+H)-M(T)$. Метод, лежащий в основе доказательства ряда лемм, приводимых ниже, был разработан А. Сельбергом в работе [1]. Здесь мы сушественно пользуемся этим методом. Определим функции $\Lambda_{z}(n)$ и $\sigma_{z, t}$, введенные А. Сельбергом. Пусть $z \geqslant 2$. Тогда

$$
\Lambda_{z}(n)= \begin{cases}\Lambda(n), & \text { если } 1 \leqslant n \leqslant z, \\ \Lambda(n) \frac{\ln ^{2}\left(z^{3} / n\right)-2 \ln ^{2}\left(z^{2} / n\right)}{2 \ln ^{2} z}, & \text { если } z<n \leqslant z^{2}, \\ \Lambda(n) \frac{\ln ^{2}\left(z^{3} / n\right)}{2 \ln ^{2} z}, & \text { если } z^{2}<n \leqslant z^{3},\end{cases}
$$

где $\Lambda(n)$ - функция Мангольдта, равная $\log p$, если $n=p^{k}, p$ - простое, $k \geqslant 1$ целое, и равная нулю в остальных случаях.

Пусть $t^{2} \geqslant z$, и пусть $\varrho=\beta+i \gamma$ пробегает значения нетривиальных нулей дзета-функции Римана с условием

$$
|t-\gamma| \leqslant z^{3|\beta-0.5|} \ln ^{-1} z
$$

Символом $\sigma_{z, t}$ обозначим следующую величину:

$$
\sigma_{z, t}=0.5+2 \max \left(|\beta-0.5|, 2 \ln ^{-1} z\right)
$$

Если гипотеза Римана верна, то $\sigma_{z, t}=0.5+4 \ln ^{-1} z$.

Лемма 13. Пусть $t \geqslant 2,2 \leqslant z \leqslant t^{2}$. Тогда имеет место равенство

$$
\begin{aligned}
S(t)=- & \frac{1}{\pi} \sum_{1<n<z^{3}} \frac{\Lambda_{z}(n)}{n^{\sigma_{z, t}}} \frac{\sin (t \ln n)}{\ln n} \\
& +O\left(\left(\sigma_{z, t}-0.5\right)\left|\sum_{1<n<z^{3}} \frac{\Lambda_{z}(n)}{n^{\sigma_{z, t}+i t}}\right|\right)+O\left(\left(\sigma_{z, t}-0.5\right) \ln t\right) .
\end{aligned}
$$

Это утверждение о приближении $S(t)$ отрезком ряда Дирихле специального вида принадлежит А. Сельбергу (см. [1]).

Лемма 14. Пусть $k \geqslant 1,0 \leqslant \nu \leqslant 4 k-$ иелье числа, $z \geqslant 2, \quad 1 \leqslant y \leqslant z^{4 k}$, $z^{3} y^{2} \leqslant x$. Тогда для всех $T$ из множсества $\mathscr{E}_{1}$ справедлива оценка

$$
\int_{T}^{T+H}\left(\sigma_{z, t}-0.5\right)^{\nu} y^{\sigma_{z, t}-0.5} d t \ll(e \sqrt{2})^{16 k} H\left((\ln z)^{-\nu}+\nu ! \frac{\ln x}{\ln z}(\ln x)^{-\nu}\right) .
$$


ДокАЗАТЕЛЬСтво. Будем следовать рассуждениям работы [1]. Обозначим символом $\psi(t)$ подынтегральную функцию. Точку $t$ промежутка $(T, T+H]$ отнесем ко множеству $A_{1}$, если для всех нулей $\varrho=\beta+i \gamma$ дзета-функции, удовлетворяюших условию

$$
|t-\gamma| \leqslant z^{3|\beta-0.5|} \ln ^{-1} z,
$$

вьполнены неравенства $|\beta-0.5| \leqslant 2 \ln ^{-1} z$. В противном случае точку $t$ отнесем ко множеству $A_{2}$. Таким образом получим

$$
\int_{T}^{T+H} \psi(t) d t=\left(\int_{A_{1}}+\int_{A_{2}}\right) \psi(t) d t=I_{1}+I_{2}
$$

Если $t \in A_{1}$, то

$$
\begin{gathered}
\sigma_{z, t}=0.5+4 \ln ^{-1} z \\
\psi(t)=\left(4 \ln ^{-1} z\right)^{\nu} y^{4 / \ln z} \leqslant(e \sqrt{2})^{16 k}(\ln z)^{-\nu},
\end{gathered}
$$

и поэтому

$$
I_{1} \leqslant \int_{T}^{T+H}(e \sqrt{2})^{16 k}(\ln z)^{-\nu} d t=(e \sqrt{2})^{16 k} H(\ln z)^{-\nu} .
$$

Если же $t \in A_{2}$, то

$$
\sigma_{z, t}=0.5+|\beta-0.5| \text { и } \psi(t)=|\beta-0.5|^{\nu} y^{2|\beta-0.5|},
$$

где $\varrho=\beta+i \gamma-$ некоторый нуль $\zeta(s)$ с условием $|t-\gamma| \leqslant z^{3|\beta-0.5|} \ln ^{-1} z$. Так как $z^{3} \leqslant z^{3} y^{2} \leqslant x$, то

$$
z^{3|\beta-0.5|} \ln ^{-1} z \leqslant z^{1.5}<\sqrt{x}<H,
$$

так что мнимая часть этого нуля отстоит от $t$ на расстояние, не превосходящее $H$, и содержится в промежутке $(T-H, T+2 H]$. Поэтому

$$
\begin{aligned}
I_{2} & \leqslant \sum_{T-H<\gamma \leqslant T+2 H}|\beta-0.5|^{\nu} y^{2|\beta-0.5|} z^{3|\beta-0.5|} \ln ^{-1} z \\
& \leqslant 2 \sum_{T-2 H<\gamma \leqslant T+2 H}^{\prime}(\beta-0.5)^{\nu}\left(z^{3} y^{2}\right)^{\beta-0.5} \ln ^{-1} z,
\end{aligned}
$$

где штрих означает суммирование по всем нулям $\varrho=\beta+i \gamma$, для которых $\beta \geqslant 0.5$ и $T-2 H<\gamma \leqslant T+2 H$. Воспользовавшись тождеством

$$
\begin{aligned}
& (\beta-0.5)^{\nu}\left(z^{3} y^{2}\right)^{\beta-0.5} \ln ^{-1} z= \\
& =\int_{0.5}^{\beta}\left(\frac{\ln z^{3} y^{2}}{\ln z}(u-0.5)^{\nu}+\frac{\nu}{\ln z}(u-0.5)^{\nu-1}\right)\left(z^{3} y^{2}\right)^{u-0.5} d u
\end{aligned}
$$


которое устанавливается интегрированием по частям, находим

$$
\begin{aligned}
I_{2} \leqslant & 2 \sum_{T-2 H<\gamma \leqslant T+2 H}^{\prime} \int_{0.5}^{\beta}\left(\frac{\ln z^{3} y^{2}}{\ln z}(u-0.5)^{\nu}+\frac{\nu}{\ln z}(u-0.5)^{\nu-1}\right)\left(z^{3} y^{2}\right)^{u-0.5} d u \\
= & 2 \sum_{T-2 H<\gamma \leqslant T+2 H} \int_{0.5}^{1} g(\varrho ; u)\left(\frac{\ln z^{3} y^{2}}{\ln z}(u-0.5)^{\nu}\right. \\
& \left.+\frac{\nu}{\ln z}(u-0.5)^{\nu-1}\right)\left(z^{3} y^{2}\right)^{u-0.5} d u \\
= & \int_{0.5}^{1}\left(\sum_{T-2 H<\gamma \leqslant T+2 H} g(\varrho ; u)\right) \\
& \times\left(\frac{\ln z^{3} y^{2}}{\ln z}(u-0.5)^{\nu}+\frac{\nu}{\ln z}(u-0.5)^{\nu-1}\right)\left(z^{3} y^{2}\right)^{u-0.5} d u
\end{aligned}
$$

где

$$
g(\varrho ; u)=g(\beta+i \gamma ; u)= \begin{cases}1, & \text { если } 0.5 \leqslant u \leqslant \beta \\ 0, & \text { если } \beta<u \leqslant 1\end{cases}
$$

Заметим теперь, что

$$
\sum_{T-2 H<\gamma \leqslant T+2 H} g(\varrho ; u)=N(u, T+2 H)-N(u, T-2 H) .
$$

Воспользовавшись утверждением теоремы А наряду с неравенством $z^{3} y^{2} \leqslant x$, получим

$$
I_{2} \ll H(\ln X) \int_{0.5}^{1} x^{-(u-0.5)}\left(\frac{\ln x}{\ln z}(u-0.5)^{\nu}+\frac{\nu}{\ln z}(u-0.5)^{\nu-1}\right) d u
$$

Положим $v=(u-0.5) \ln x$. Тогда оценка для $I_{2}$ примет вид

$$
\begin{aligned}
I_{2} & \ll H L(\ln z)^{-1} \int_{0}^{0.5 \ln x} e^{-v}\left(\ln x\left(\frac{v}{\ln x}\right)^{\nu}+\nu\left(\frac{v}{\ln x}\right)^{\nu-1}\right) \frac{d v}{\ln x} \\
& \ll H \varepsilon^{-1} \frac{(\ln x)^{1-\nu}}{\ln z} \int_{0}^{+\infty} e^{-v}\left(v^{\nu}+\nu v^{\nu-1}\right) d v \ll \Gamma(1+\nu) H \frac{(\ln x)^{1-\nu}}{\ln z} .
\end{aligned}
$$

Используя оценки для $I_{1}$ и $I_{2}$, находим

$$
\int_{T}^{T+H} \psi(t) d t \ll(e \sqrt{2})^{16 k} H\left((\ln z)^{-\nu}+\nu ! \frac{\ln x}{\ln z}(\ln x)^{-\nu}\right) .
$$

Лемма доказана. 
Лемма 15 [4, лемма 3]. Пусть $H \geqslant 10, k$ - челое число, $k \geqslant 1,1<$ $y \leqslant H^{1 / k}$, и пусть $а(p), a^{\prime}(p)$ - произвольные комплекснозначные функиии, удовлетворяющие при $p \leqslant y$ неравенствам

$$
|a(p)| \leqslant c \frac{\ln p}{\ln y}, \quad\left|a^{\prime}(p)\right| \leqslant c .
$$

Тогда для интегралов

$$
I=\int_{0}^{H}\left|\sum_{p<y} a(p) p^{-0.5-i t}\right|^{2 k} d t
$$

$u$

$$
I^{\prime}=\int_{0}^{H}\left|\sum_{p<y} a^{\prime}(p) p^{-1-2 i t}\right|^{2 k} d t
$$

имеют место оченки

$$
I \leqslant\left(c_{1} c^{2} k\right)^{k} H, \quad I^{\prime} \leqslant\left(c_{1} c^{2} k\right)^{k} H,
$$

әде $c_{1}>0-$ абсолютная постоянная.

Лемма 16. Пусть $k$ - иелое число, $1 \leqslant k \leqslant(\ln \ln X)^{0.5}, H^{1 / 20 k}<y \leqslant H^{1 / k}$. Тогда для любого Т из множества $\mathscr{E}_{1}$ справедливо неравенство

$$
\int_{T}^{T+H}\left|S(t)+\frac{1}{\pi} \sum_{p<y} \frac{\sin (t \ln p)}{\sqrt{p}}\right|^{2 k} d t \leqslant(c k)^{2 k} H
$$

ЗАмЕчАниЕ. Доказательство этого утверждения основано на двух предыдуших леммах и проводится с использованием методов и приемов работ [1], [4] и [5]. Равномерная по $k$ оценка подобного интеграла была получена А. Гошем [4] для случая $H=T^{0.5+\alpha}$ и улучшена впоследствии К. Тсангом [18].

ДоКАЗАТЕЛЬСТВо. Возьмем в лемме $13 z=H^{1 /(60 k)}$ и прибавим к обеим частям получившегося равенства сумму

$$
\frac{1}{\pi} \sum_{p<z^{3}} \frac{\sin (t \ln p)}{\sqrt{p}}
$$

Переходя к оценкам, получим

$$
S(t)+\frac{1}{\pi} \sum_{p<z^{3}} \frac{\sin (t \ln p)}{\sqrt{p}} \ll \sum_{j=1}^{7} R_{j}
$$


где

$$
\begin{array}{ll}
R_{1}=\left|\sum_{p<z^{3}} \frac{\Lambda(p)-\Lambda_{z}(p)}{\sqrt{p} \ln p} p^{-i t}\right|, & R_{2}=\left|\sum_{p<z^{3}} \frac{\Lambda_{z}(p)}{\sqrt{p} \ln p}\left(1-p^{0.5-\sigma_{z, t}}\right) p^{-i t}\right|, \\
R_{3}=\left(\sigma_{z, t}-0.5\right)\left|\sum_{p<z^{3}} \frac{\Lambda_{z}(p)}{p^{\sigma_{z, t}+i t}}\right|, & R_{4}=\left|\sum_{p<z^{1.5}} \frac{\Lambda_{z}\left(p^{2}\right)}{p \ln p} p^{-2 i t}\right|, \\
R_{5}=\left|\sum_{p<z^{1.5}} \frac{\Lambda_{z}\left(p^{2}\right)}{p \ln p}\left(1-p^{1-2 \sigma_{z, t}}\right) p^{-2 i t}\right|, & R_{6}=\left(\sigma_{z, t}-0.5\right)\left|\sum_{p<z^{1.5}} \frac{\Lambda_{z}\left(p^{2}\right)}{p^{2 \sigma_{z, t}+2 i t}}\right|, \\
R_{7}=\sum_{r>2} \sum_{p^{r}<z^{3}} \frac{\ln p}{p^{r / 2}} . &
\end{array}
$$

Преобразуем величины $R_{j}$ для $j=2,5,6$ и 7 . Прежде всего заметим, что

$$
R_{7} \ll 1 \ll\left(\sigma_{z, t}-0.5\right) \ln X, \quad R_{6} \ll\left(\sigma_{z, t}-0.5\right) \sum_{p<z^{1.5}} \frac{\ln p}{p} \ll\left(\sigma_{z, t}-0.5\right) \ln X .
$$

По теореме Лагранжа о конечных приращениях

$$
0 \leqslant 1-p^{1-2 \sigma_{z, t}}=\left(2 \sigma_{z, t}-1\right)(\ln p) p^{\theta\left(1-2 \sigma_{z, t}\right)} \leqslant 2\left(\sigma_{z, t}-0.5\right) \ln p .
$$

Поэтому

$$
R_{5} \leqslant \sum_{p<z^{1.5}} \frac{\Lambda_{z}\left(p^{2}\right)}{p \ln p}\left(2 \sigma_{z, t}-1\right) \ln p \ll\left(\sigma_{z, t}-0.5\right) \sum_{p<z^{1.5}} \frac{\ln p}{p} \ll\left(\sigma_{z, t}-0.5\right) \ln X .
$$

Кроме того, из тождества

$$
\frac{1-p^{0.5-\sigma_{z, t}}}{\sqrt{p} \ln p}=\int_{0.5}^{\sigma_{z, t}} p^{-u} d u
$$

следует неравенство

$$
R_{2}=\left|\sum_{p<z^{3}} \Lambda_{z}(p) \int_{0.5}^{\sigma_{z, t}} p^{-u-i t} d u\right| \leqslant \int_{0.5}^{\sigma_{z, t}}\left|\sum_{p<z^{3}} \frac{\Lambda_{z}(p)}{p^{u+i t}}\right| d u .
$$

Если $0.5 \leqslant u \leqslant \sigma_{z, t}$, то

$$
\left|\sum_{p<z^{3}} \frac{\Lambda_{z}(p)}{p^{u+i t}}\right|=z^{u-0.5}\left|\int_{u}^{+\infty} z^{0.5-v} \sum_{p<z^{3}} \frac{\Lambda_{z}(p) \ln (z p)}{p^{v+i t}} d v\right| .
$$

Поэтому неравенство для $R_{2}$ принимает вид

$$
R_{2} \leqslant\left(\sigma_{z, t}-0.5\right) z^{\sigma_{z, t}-0.5} \int_{0.5}^{+\infty} z^{0.5-v}\left|\sum_{p<z^{3}} \frac{\Lambda_{z}(p) \ln (z p)}{p^{v+i t}}\right| d v
$$


Переходя к оценке интеграла из условия леммы и пользуясь неравенством Гёльдера, заключаем, что

$$
\int_{T}^{T+H}\left|S(t)+\frac{1}{\pi} \sum_{p<z^{3}} \frac{\sin (t \ln p)}{\sqrt{p}}\right|^{2 k} d t \ll 2^{4 k} \sum_{j=1}^{4} K_{j}
$$

где

$$
\begin{gathered}
K_{1}=\int_{T}^{T+H}\left|\sum_{p<z^{3}} \frac{\Lambda(p)-\Lambda_{z}(p)}{\sqrt{p} \ln p}\right|^{2 k} d t \\
K_{2}=\int_{T}^{T+H}\left|\sum_{p<z^{1.5}} \frac{\Lambda_{z}\left(p^{2}\right)}{p \ln p} p^{-2 i t}\right|^{2 k} d t, \quad K_{3}=(\ln X)^{2 k} \int_{T}^{T+H}\left(\sigma_{z, t}-0.5\right)^{2 k} d t
\end{gathered}
$$

и

$$
K_{4}=\int_{T}^{T+H}\left(\sigma_{z, t}-0.5\right)^{2 k} z^{2 k\left(\sigma_{z, t}-0.5\right)}\left(\int_{0.5}^{+\infty} z^{0.5-v}\left|\sum_{p<z^{3}} \frac{\Lambda_{z}(p) \log (z p)}{p^{u+i t}} d u\right|\right)^{2 k} d t .
$$

Полагая последовательно

$$
\begin{aligned}
& y=z^{3}, \quad a(p)=\frac{\Lambda(p)-\Lambda_{z}(p)}{\ln p} p^{-i T} \\
& y=z^{1.5}, \quad a^{\prime}(p)=\frac{\Lambda_{z}\left(p^{2}\right)}{\ln p} p^{-2 i T}
\end{aligned}
$$

с помощью леммы 15 убеждаемся в том, что

$$
K_{j} \ll(c k)^{k} H, \quad j=1,2 .
$$

Взяв в лемме $14 y=1, \nu=2 k$, приходим к оценке

$$
\begin{aligned}
K_{3} & \ll(\ln X)^{2 k}(e \sqrt{2})^{16 k} H\left(\left(\ln H^{1 /(60 k)}\right)^{-2 k}+(2 k) ! \frac{(\ln x)^{1-2 k}}{\ln H^{1 /(60 k)}}\right) \\
& \ll c^{k}(\ln X)^{2 k} H\left(k^{2 k}(\ln X)^{-2 k}+k^{2 k}(\ln X)^{-2 k}\right) \ll\left(c_{1} k\right)^{2 k} H .
\end{aligned}
$$

Наконец, применяя к величине $K_{4}$ неравенство Коши, получаем

$$
K_{4} \ll \sqrt{K_{5} K_{6}},
$$

где

$$
\begin{aligned}
& K_{5}=\int_{T}^{T+H}\left(\sigma_{z, t}-0.5\right)^{4 k} z^{4 k\left(\sigma_{z, t}-0.5\right)} d t \\
& K_{6}=\int_{T}^{T+H}\left(\int_{0.5}^{+\infty} z^{0.5-u}\left|\sum_{p<z^{3}} \frac{\Lambda_{z}(p) \log (z p)}{p^{u+i t}}\right| d u\right)^{4 k} d t
\end{aligned}
$$


Величину $K_{5}$ оценим, пользуясь леммой 14 при $y=x^{4 k}, \nu=4 k$ :

$K_{5} \ll(e \sqrt{2})^{16 k} H\left(\left(\ln H^{1 /(60 k)}\right)^{-4 k}+(4 k) ! \frac{(\ln x)^{1-4 k}}{\ln H^{1 /(60 k)}}\right) \ll(c k)^{4 k} H(\ln X)^{-4 k}$.

Интеграл $K_{6}$ оценим с помошью неравенства Гёльдера:

$$
\begin{aligned}
K_{6} & \ll \int_{T}^{T+H}\left(\int_{0.5}^{+\infty} z^{0.5-u} d u\right)^{4 k-1} \int_{0.5}^{+\infty} z^{0.5-u}\left|\sum_{p<z^{3}} \frac{\Lambda_{z}(p) \log (z p)}{p^{u+i t}}\right|^{4 k} d u d t \\
& \ll(\ln z)^{1-4 k} \int_{0.5}^{+\infty} z^{0.5-u} \int_{T}^{T+H}\left|\sum_{p<z^{3}} \frac{\Lambda_{z}(p) \log (z p)}{p^{u+i t}}\right|^{4 k} d t d u \\
& \ll(\ln z)^{4 k+1} \int_{0.5}^{+\infty} z^{0.5-u} \int_{T}^{T+H}\left|\sum_{p<z^{3}} \frac{a(p)}{\sqrt{p}} p^{-i t}\right|^{4 k} d t d u,
\end{aligned}
$$

где

$$
a(p)=\frac{\Lambda_{z}(p) \ln (z p)}{p^{u-0.5+i T} \ln ^{2} z} .
$$

Неравенство леммы 15 , примененное к интегралу по $t$, приводит к оценке

$$
K_{6} \ll(c k)^{2 k} H(\ln z)^{4 k} \ll(c k)^{2 k} H\left(k^{-1} \ln X\right)^{4 k} \ll\left(c_{1} k\right)^{-2 k} H(\ln X)^{4 k} .
$$

Оценивая интеграл $K_{4}$, будем иметь

$$
K_{4} \ll \sqrt{K_{5}} \sqrt{K_{6}} \ll(c k)^{2 k} \sqrt{H}(\ln X)^{-2 k}\left(c_{1} k\right)^{-k} \sqrt{H}(\ln X)^{2 k} \ll\left(c_{2} k\right)^{k} H .
$$

Таким образом,

$$
\int_{T}^{T+H}\left|S(t)+\frac{1}{\pi} \sum_{p<z^{3}} \frac{\sin (t \ln p)}{\sqrt{p}}\right|^{2 k} d t \leqslant(c k)^{2 k} H .
$$

Далее,

$$
\begin{aligned}
\int_{T}^{T+H} \mid S(t) & +\left.\frac{1}{\pi}\left(\sum_{p<z^{3}}+\sum_{z^{3} \leqslant p<y}\right) \frac{\sin (t \ln p)}{\sqrt{p}}\right|^{2 k} d t \\
\leqslant & 2^{2 k}\left(\int_{T}^{T+H}\left|S(t)+\frac{1}{\pi} \sum_{p<z^{3}} \frac{\sin (t \ln p)}{\sqrt{p}}\right|^{2 k} d t\right. \\
+ & \left.\int_{T}^{T+H}\left|\sum_{z^{3} \leqslant p<y} \frac{\sin (t \ln p)}{\sqrt{p}}\right|^{2 k} d t\right) .
\end{aligned}
$$

По доказанному вьше первый из интегралов не превосходит величины $(c k)^{2 k} H$, второй интеграл оценивается сверху величиной

$$
\int_{T}^{T+H}\left|\sum_{z^{3} \leqslant p<y} p^{-0.5-i t}\right|^{2 k} d t=\int_{T}^{T+H}\left|\sum_{p<y} \frac{a(p)}{\sqrt{p}} p^{-i t}\right|^{2 k} d t
$$


где

Поскольку

$$
a(p)=\left\{\begin{array}{l}
0, \text { если } p<z^{3} \\
1, \text { если } z^{3} \leqslant p<y .
\end{array}\right.
$$

$$
|a(p)| \leqslant \frac{\ln y}{\ln p} \frac{\ln p}{\ln y} \leqslant \frac{\ln H^{1 / k}}{\ln H^{1 /(20 k)}} \frac{\ln p}{\ln y}=20 \frac{\ln p}{\ln y},
$$

то по лемме 15 последний интеграл есть $O\left((c k)^{k} H\right)$. Это и завершает доказательство леммы.

ЛЕмма 17. Пусть $y>e^{3}, H>0, \delta>0, k \geqslant 1, \mu \geqslant 0$ - иелье числа, $\mu+\nu=2 k, \quad a(n)$ - последовательность комплексных чисел такая, что $|a(n)| \leqslant \delta$ для всех $n \leqslant y$, и пусть $p_{1}, \ldots, p_{\mu}, q_{1}, \ldots, q_{\nu}$ пробегают значения простых чисел промежсткка $(1, y], p_{1} \ldots p_{\mu} \neq q_{1} \ldots q_{\nu}$. Тогда для интеграла

$$
I=\int_{0}^{H} \sum_{p_{1}, \ldots, p_{\mu}} \sum_{q_{1}, \ldots, q_{\nu}} \frac{a\left(p_{1}\right) \ldots a\left(p_{\mu}\right) \bar{a}\left(q_{1}\right) \ldots \bar{a}\left(q_{\nu}\right)}{\left(p_{1} \ldots q_{\nu}\right)^{0.5}}\left(\frac{q_{1} \ldots q_{\nu}}{p_{1} \ldots p_{\mu}}\right)^{i t} d t
$$

справедлива оченка

$$
|I| \leqslant 2 \delta^{2 k} y^{3 k}
$$

ДокАЗАТЕЛЬСтво. Положим для краткости $P=p_{1} \ldots p_{\mu}, Q=q_{1} \ldots q_{\nu}$. Производя интегрирование, получим

$$
|I|=\left|\sum_{P \neq Q} \frac{a\left(p_{1}\right) \ldots \bar{a}\left(q_{\nu}\right)}{\sqrt{P Q}} \frac{\left(Q P^{-1}\right)^{i H}-1}{i \ln \left(Q P^{-1}\right)}\right| \leqslant 2 \delta^{2 k} \sum_{P \neq Q} \frac{\left|\ln \left(Q P^{-1}\right)\right|^{-1}}{\sqrt{P Q}} .
$$

Поскольку $\left|\ln \left(Q P^{-1}\right)\right|^{-1} \leqslant \max (Q, P) \leqslant y^{2 k}$, то приходим к цепочке неравенств

$$
\begin{aligned}
|I| & \leqslant 2(\delta y)^{2 k} \sum_{P, Q} \frac{1}{\sqrt{P Q}} \leqslant 2(\delta y)^{2 k}\left(\sum_{p \leqslant y} \frac{1}{\sqrt{p}}\right)^{2 k} \\
& \leqslant 2(\delta y)^{2 k}\left(\frac{3 \sqrt{y}}{\ln y}\right)^{2 k}<2 \delta^{2 k} y^{3 k}
\end{aligned}
$$

которые и завершают доказательство леммы.

ТЕОРЕМА 1. Пусть $k$ - челое число, удовлетворяющее условиям

$$
1 \leqslant k \leqslant(\ln \ln X)^{0.5}, \quad 3 k \varepsilon^{-1}(\ln X)^{-1}<h \leqslant(\ln X)^{-0.5},
$$

u nycmb

$$
I(2 k)=\int_{T}^{T+H}|S(t)|^{2 k} d t, \quad J(2 k)=\int_{T}^{T+H}\left|S_{1}(t+h)-S_{1}(t)\right|^{2 k} d t .
$$

Тогда для любого Т из множества $\mathscr{E}_{1}$ справедливы следующие асимптотические формуль:

$$
\begin{aligned}
& I(2 k)=\frac{(2 k) !}{k !} \frac{H}{(2 \pi)^{2 k}}(\ln \ln T)^{k}(1+O(R)), \\
& J(2 k)=\frac{(2 k) !}{k !}\left(\frac{h}{2 \pi}\right)^{2 k}\left(\ln \left(h^{-1}\right)\right)^{k}(1+O(R)),
\end{aligned}
$$

əде $R=c^{k}(\ln \ln X)^{-0.5}$. 
ДоКАЗАТЕЛЬСТВО. Положим $y=H^{1 /(3 k)}$,

$$
W(t)=\frac{1}{\pi} \sum_{p<y} \frac{\sin (t \ln p)}{\sqrt{p}}, \quad R(t)=S(t)+W(t),
$$

и представим первый из интегралов в виде

$$
I(2 k)=I_{1}+I_{2}
$$

где

$$
I_{1}=\int_{T}^{T+H} W^{2 k}(t) d t, \quad I_{2}=\int_{T}^{T+H} \sum_{\nu=1}^{2 k}\left(\begin{array}{c}
2 k \\
\nu
\end{array}\right) W^{2 k-\nu}(t) R^{\nu}(t) d t .
$$

Если положить теперь $W=\left(W_{1}-\bar{W}_{1}\right) / 2$, где

$$
W_{1}=\frac{i}{2 \pi} \sum_{p<y} p^{-0.5-i t},
$$

то $I_{1}$ запишется в виде суммы

$$
I_{1}=I_{3}+I_{4}
$$

где

$$
\begin{aligned}
I_{3} & =(2 \pi)^{-2 k}\left(\begin{array}{c}
2 k \\
k
\end{array}\right) \int_{T}^{T+H}\left|W_{1}(t)\right|^{2 k} d t \\
I_{4} & =(2 \pi)^{-2 k} \sum_{\nu \neq k}(-1)^{k+\nu}\left(\begin{array}{c}
2 k \\
\nu
\end{array}\right) \int_{T}^{T+H} W_{1}^{\nu} \bar{W}_{1}^{2 k-\nu} d t .
\end{aligned}
$$

Применяя к каждому из интегралов

$$
\int_{T}^{T+H} W_{1}^{\nu} \bar{W}_{1}^{2 k-\nu} d t, \quad \nu \neq k
$$

оценку леммы $17 \mathrm{c} \delta=1$, получим

$$
\left|I_{4}\right| \leqslant(2 \pi)^{-2 k} \sum_{\nu \neq k} 2 y^{3 k}\left(\begin{array}{c}
2 k \\
\nu
\end{array}\right)<2 \pi^{-2 k} y^{3 k}<H .
$$

Раскрывая модуль в $I_{3}$ и применяя к внедиагональным слагаемым лемму 17 , приходим к равенству

$$
I_{3}=(2 \pi)^{-2 k}\left(\begin{array}{c}
2 k \\
k
\end{array}\right) H \sum_{p_{1}, \ldots, q_{k}<y}^{\prime}\left(p_{1} \ldots q_{k}\right)^{-0.5}+\theta H,
$$

где штрих означает суммирование по числам $p_{1}, \ldots, q_{k}$, для которых $p_{1} \ldots p_{k}=$ $q_{1} \ldots q_{k}$. Последняя сумма вычислена в работах [4] и [14]. Воспользуемся готовой формулой из [4], в которой оценка остаточного члена является равномерной по $k$ :

$$
\sum_{p_{1}, \ldots, q_{k}<y}^{\prime}\left(p_{1} \ldots q_{k}\right)^{-0.5}=k !(\ln \ln T)^{k}+O\left((c k)^{k}(\ln \ln T)^{k-1}\right) .
$$


Таким образом,

$$
I_{1}=k !(\ln \ln T)^{k}\left(1+O\left(c^{k} \ln \ln X\right)^{-1}\right),
$$

так что $I_{1} \leqslant\left(c_{1} k\right)^{k} H(\ln \ln T)^{k}$. Переходя к оценке $I_{2}$, будем иметь

$$
\begin{aligned}
I_{2} & \leqslant \int_{T}^{T+H} \sum_{\nu=1}^{2 k}\left(\begin{array}{c}
2 k \\
\nu
\end{array}\right)|W(t)|^{2 k-\nu}|R(t)|^{\nu} d t \\
& \leqslant 2 k \int_{T}^{T+H}|R(t)|(|W(t)|+|R(t)|)^{2 k-1} d t \leqslant 2^{2 k} k\left(I_{5}+I_{6}\right),
\end{aligned}
$$

где

$$
I_{5}=\int_{T}^{T+H}|R(t)|^{2 k} d t, \quad I_{6}=\int_{T}^{T+H}|R(t)||W(t)|^{2 k-1} d t .
$$

Поскольку $k \leqslant(\ln \ln X)^{0.5}$, то $k^{2 k} \leqslant k^{k}(\ln \ln X)^{k / 2} \leqslant k^{k}(\ln \ln X)^{k-0.5}$ и по лемме 16

$$
I_{5} \leqslant(c k)^{2 k} H \leqslant\left(c_{1} k\right)^{k} H(\ln \ln X)^{k-0.5} .
$$

Для оценки $I_{6}$ воспользуемся неравенством Гёльдера:

$$
I_{6} \leqslant I_{1}^{1-1 /(2 k)} I_{5}^{1 /(2 k)} \leqslant\left(c_{3} k\right)^{k} H(\ln \ln X)^{k-0.5} .
$$

Таким образом,

$$
\left|I_{2}\right| \leqslant\left(c_{4} k\right)^{k} H(\ln \ln X)^{k-0.5} .
$$

Из последнего неравенства следует асимптотическая формула для $I(2 k)$.

Обратимся теперь к вычислению $J(2 k)$. Поскольку

$$
S_{1}(t+h)-S_{1}(t)=-\int_{0}^{h} W(t+u) d u+\int_{0}^{h} R(t+u) d u,
$$

то интеграл $J(2 k)$ представится в виде суммы

$$
J(2 k)=J_{1}+J_{2}
$$

где

$$
\begin{aligned}
& J_{1}=\int_{T}^{T+H}\left(\int_{0}^{h} W(t+u) d u\right)^{2 k} d t \\
& J_{2}=\int_{T}^{T+H} \sum_{\nu=1}^{2 k}(-1)^{\nu}\left(\begin{array}{c}
2 k \\
\nu
\end{array}\right)\left(\int_{0}^{h} W(t+u) d u\right)^{2 k-\nu}\left(\int_{0}^{h} R(t+u) d u\right)^{\nu} d t .
\end{aligned}
$$

Интегрируя по $u$, находим

$$
\int_{0}^{h} W(t+u) d u=\frac{1}{2 \pi}(V(t)+\bar{V}(t))
$$


где

$$
V(t)=\sum_{p<y} \frac{a(p)}{\sqrt{p}} p^{i t}, \quad a(p)=\frac{1-p^{i h}}{\ln p} .
$$

Пользуясь леммой 17 и тем, что $|a(p)|=2|\sin (0.5 h \ln p)| \ln ^{-1} p \leqslant h$, заключаем, что

$$
J_{1}=(2 \pi)^{-2 k}\left(\begin{array}{c}
2 k \\
k
\end{array}\right) H \sum_{p_{1}, \ldots, q_{k}}^{\prime} \frac{a\left(p_{1}\right) \ldots \bar{a}\left(q_{k}\right)}{\left(p_{1} \ldots q_{k}\right)^{0.5}}+\theta H h^{2 k}
$$

где штрих означает суммирование по наборам $p_{1}, \ldots, q_{k}$, для которых $p_{1} \ldots p_{k}=$ $q_{1} \ldots q_{k}$. Воспользуемся готовым выражением для последней суммы, которое получено в [4]:

$$
\sum_{p_{1}, \ldots, q_{k}}^{\prime} \frac{a\left(p_{1}\right) \ldots \bar{a}\left(q_{k}\right)}{\left(p_{1} \ldots q_{k}\right)^{0.5}}=k !\left(\sum_{p<y} \frac{4 \sin ^{2}(0.5 h \ln p)}{p \ln ^{2} p}\right)^{k}+\theta(c k)^{k} H h^{2 k}(\ln \ln X)^{k-2} .
$$

Преобразуем теперь сумму по $p<y$. Так как $h>3 k \varepsilon^{-1}(\ln X)^{-1}$, то

$$
\exp (1 / h)<\exp \left((3 k)^{-1} \varepsilon \ln X\right)=H^{1 /(3 k)}=y .
$$

Если $p<\exp \left(h^{-1}\right)$, то $0.5 h \ln p \leqslant 0.5$ и

$$
\sum_{p \leqslant e^{1 / h}} \frac{4 \sin ^{2}(0.5 h \ln p)}{p \ln ^{2} p}=\sum_{p \leqslant e^{1 / h}} \frac{h^{2}}{4 p}+\theta \sum_{p \leqslant e^{1 / h}} \frac{h^{4} \ln ^{2} p}{p}=\frac{h^{2}}{4} \ln \frac{1}{h}+O\left(h^{2}\right) .
$$

Оставшуюся часть суммы по $p>\exp \left(h^{-1}\right)$ оценим тривиально:

$$
\sum_{e^{1 / h}<p<y} \frac{4 \sin ^{2}(0.5 h \ln p)}{p \ln ^{2} p} \leqslant \sum_{p>e^{1 / h}}\left(p \ln ^{2} p\right)^{-1}=O\left(h^{2}\right) .
$$

Подставляя полученные равенства в выражение для $J_{1}$, будем иметь

$$
\begin{aligned}
J_{1} & =(2 \pi)^{-2 k}\left(\begin{array}{c}
2 k \\
k
\end{array}\right)\left(k ! H h^{2 k}\left(\ln \left(h^{-1}\right)\right)^{k}+O\left(c^{k} H h^{2 k}(\ln \ln X)^{k-1}\right)\right) \\
& =\frac{(2 k) !}{k !}\left(\frac{h}{2 \pi}\right)^{2 k} H\left(\ln \left(h^{-1}\right)\right)^{k}\left(1+O\left(c^{k}(\ln \ln X)^{-1}\right)\right) .
\end{aligned}
$$

С помощью тех же рассуждений, которые использовались при вычислении $I(2 k)$, получаем оценку

$$
J_{2} \leqslant k\left(2^{2 k}\right)\left(J_{5}+J_{1}^{1-1 /(2 k)} J_{5}^{1 /(2 k)}\right)
$$

где

$$
J_{5}=\int_{T}^{T+H}\left(\int_{0}^{h}|R(t+u)| d u\right)^{2 k} d t .
$$

Пользуясь полученными выше оценками $J_{1}$ и $J_{2}$, находим

$$
J_{2} \leqslant\left(c_{1} k\right)^{k} H h^{2 k}(\ln \ln X)^{k-0.5} .
$$

Это и завершает доказательство теоремы 1.

Асимптотические формулы теоремы 1 мы используем для нахождения первых моментов величин $S(t)$ и $S_{1}(t+h)-S_{1}(t)=\int_{0}^{h} S(t+u) d u$. В основе доказательства следующей теоремы лежит идея сведения вычисления интегралов от модуля данной функции к вычислению ряда ее четных моментов (см. [4], [15], [16, гл. I], [17]). 
Teopema 2. Пусmb

$$
(\ln \ln \ln X)^{2}(\ln X)^{-1} \leqslant h \leqslant(\ln X)^{-0.5},
$$

u nycmb

$$
I_{1}=\int_{T}^{T+H}|S(t)| d t, \quad I_{2}=\int_{T}^{T+H}\left|S_{1}(t+h)-S_{1}(t)\right| d t .
$$

Тогда для любого $T$ из множества $\mathscr{E}_{1}$ имеют место асимптотические формульь

$$
I_{1}=\pi^{-3 / 2} H \sqrt{\ln \ln T}(1+O(R)), \quad I_{2}=\pi^{-3 / 2} H h \sqrt{\ln \left(h^{-1}\right)}(1+O(R)),
$$

əде $R=(\ln \ln \ln X)^{-0.5}$.

ДокАЗАТЕЛЬСТВО. Положим для $T \leqslant t \leqslant T+H$

$$
F_{1}(t)=\frac{2 \pi S(t)}{\sqrt{\ln \ln T}}, \quad F_{2}(t)=\frac{2 \pi\left(S_{1}(t+h)-S_{1}(t)\right)}{h \sqrt{\ln \left(h^{-1}\right)}}
$$

и рассмотрим интеграл

$$
I=H^{-1} \int_{T}^{T+H}|F(t)| d t,
$$

где $F$ - одна из функций $F_{1}$ и $F_{2}$. Покажем, что для $I$ верна асимптотическая формула

$$
I=2 \pi^{-1 / 2}\left(1+O\left((\ln \ln \ln X)^{-0.5}\right)\right),
$$

из которой будут следовать выражения для $I_{1}$ и $I_{2}$. Схема доказательства теоремы такова. Рассмотрим тождество

$$
|a|=\frac{2}{\pi} \int_{0}^{+\infty}\left(\frac{\sin (a u)}{u}\right)^{2} d u
$$

справедливое для любого вешественного числа $a$. Полагая в нем $a=F(t)$ и интегрируя полученное равенство по $t$ в пределах от $t=T$ до $t=T+H$, приходим к следуюшему выражению для $I$ :

$$
I=\frac{2 H^{-1}}{\pi} \int_{T}^{T+H} \int_{0}^{+\infty}\left(\frac{\sin (|F(t)| u)}{u}\right)^{2} d u d t .
$$

Заменим промежуток $(0,+\infty)$ на конечньй и приблизим $\sin ^{2}(F(t) u)$ отрезком ряда Тейлора по четным степеням $F(t) u$. Поменяв местами порядки интегрирования, в правой части равенства получим линейную комбинацию интегралов, для которых в теореме 1 были выведены асимптотические формулы. Таким образом, вычисление первого момента $F(t)$ сводится к вычислению некоторой суммы, в которой участвуют лишь четные моменты этой функции. 
Произведем теперь необходимые вычисления. Пусть $\lambda=\lambda(T) \geqslant 2$. Тогда

$$
I=J_{1}+R_{1}
$$

где

$$
\begin{aligned}
J_{1} & =2 \pi H^{-1} \int_{0}^{\lambda} \int_{T}^{T+H}\left(\frac{\sin (|F(t)| u)}{u}\right)^{2} d u d t \\
R_{1} & =2 \pi H^{-1} \int_{\lambda}^{+\infty} \int_{T}^{T+H}\left(\frac{\sin (|F(t)| u)}{u}\right)^{2} d u d t \leqslant \int_{\lambda}^{+\infty} \frac{d u}{u^{2}}=\lambda^{-1} .
\end{aligned}
$$

Возьмем теперь целое $N$ из промежутка $\lambda \leqslant N \leqslant 0.1(\ln \ln X)^{0.5}$. По формуле Тейлора

$$
\sin ^{2}(F(t) u)=\frac{1}{2} \sum_{n=1}^{N} \frac{(-1)^{n-1}}{(2 n) !}(2 u F(t))^{2 n}+\theta \frac{(2 u F(t))^{2 N+2}}{(2 N+2) !} .
$$

Подставляя это выражение в равенство для $J_{1}$, находим

$$
J_{1}=J_{2}+R_{2}
$$

где

$$
\begin{gathered}
J_{2}=\frac{1}{\pi} \int_{0}^{\lambda} u^{-2}\left(\sum_{n=1}^{N} \frac{(-1)^{n-1}}{(2 n) !}(2 u)^{2 n} j(n)\right) d u \\
R_{2} \leqslant \frac{8}{\pi} \int_{0}^{\lambda} \frac{(2 u)^{2 N}}{(2 N+2) !} j(N+1) d u \\
j(n)=H^{-1} \int_{T}^{T+H}|F(t)|^{2 n} d t
\end{gathered}
$$

Из теоремы 1 следует, что

$$
j(n)=\frac{(2 n) !}{n !}\left(1+O\left(c^{n}(\ln \ln X)^{-0.5}\right)\right),
$$

и $j(n) \ll(c n)^{n}$, где $c=c(\varepsilon)>0$ - постоянная. Воспользовавшись последним неравенством, оценим величину $R_{2}$ :

$$
R_{2} \ll \frac{\lambda}{N} \frac{(2 \lambda)^{2 N}}{(2 N+2) !}(c(N+1))^{N+1} \ll\left(\frac{2 c e \lambda^{2}}{N}\right)^{N} \ll\left(\frac{c_{1} \lambda^{2}}{N}\right)^{N},
$$

где $c_{1}=4 c e$. Заменяя по приведенной выше формуле значения $j(n)$ в выражении для $J_{2}$, находим

$$
J_{2}=J_{3}+R_{3}
$$


где

$$
\begin{gathered}
J_{3}=\frac{1}{\pi} \int_{0}^{\lambda} u^{-2}\left(\sum_{n=1}^{N} \frac{(-1)^{n-1}}{n !}(2 u)^{2 n}\right) d u, \\
R_{3} \ll \int_{0}^{\lambda} u^{-2}\left(\sum_{n=1}^{N} \frac{u^{2 n}}{n !} \frac{c^{n}}{\sqrt{\ln \ln X}}\right) d u \\
\ll(\ln \ln X)^{-0.5} \sum_{n=1}^{N} \frac{\left(c \lambda^{2}\right)^{n}}{n !} \ll e^{c \lambda^{2}}(\ln \ln X)^{-0.5} .
\end{gathered}
$$

Поскольку

$$
\sum_{n=1}^{N} \frac{(-1)^{n-1}}{n !}(2 u)^{2 n}=1-e^{-4 u^{2}}+\theta \frac{(2 u)^{2 N+2}}{(N+1) !},
$$

то интеграл $I_{3}$ принимает вид

$$
I_{3}=I_{4}+R_{4}
$$

где

$$
\begin{gathered}
I_{4}=\pi^{-1} \int_{0}^{+\infty} u^{-2}\left(1-e^{-4 u^{2}}\right) d u \\
R_{4}=-\pi^{-1} \int_{\lambda}^{+\infty} u^{-2}\left(1-e^{-4 u^{2}}\right) d u+4 \theta \pi^{-1} \int_{0}^{+\infty} \frac{(2 u)^{2 N}}{(N+1) !} d u \\
\ll \lambda^{-1}+\lambda N^{-2.5}\left(\frac{4 e \lambda^{2}}{N}\right)^{N} \ll \lambda^{-1}+\left(\frac{c_{1} \lambda^{2}}{N}\right)^{N} .
\end{gathered}
$$

Значение $I_{4}$ находим интегрированием по частям:

$$
\begin{aligned}
I_{4} & =\pi^{-1} \int_{0}^{+\infty} v^{-3 / 2}\left(1-e^{-v}\right) d v=\pi^{-1} \int_{0}^{+\infty}\left(1-e^{-v}\right) d\left(-2 v^{-1 / 2}\right) \\
& =2 \pi^{-1} \int_{0}^{+\infty} v^{-1 / 2} e^{-v} d v=2 \pi^{-1 / 2} .
\end{aligned}
$$

Используя оценки величин $R_{j}, j=1,2,3,4$, приходим к равенству

$$
I=2 \pi^{-1 / 2}(1+O(R)),
$$

где

$$
R=\lambda^{-1}+\left(\frac{c_{1} \lambda^{2}}{N}\right)^{N}+e^{c \lambda^{2}}(\ln \ln X)^{-0.5} .
$$

Возьмем теперь $N=[0.5 \ln \ln \ln T]+1, \lambda=0.5\left(c_{1}^{-1} \ln \ln \ln T\right)^{0.5}$. Тогда

$$
\left(\frac{c_{1} \lambda^{2}}{N}\right)^{N} \ll 2^{-N} \ll(\ln \ln X)^{-0.3}
$$

и

$$
e^{c \lambda^{2}}(\ln \ln X)^{-0.5} \ll \exp \left(c_{1} \cdot 0.25 c_{1}^{-1}-0.5\right) \ln \ln \ln X \ll(\ln \ln X)^{-0.25} .
$$

Таким образом, $R \ll \lambda^{-1} \ll(\ln \ln \ln X)^{-0.25}$. Полагая в формуле для $I$ последовательно $F=F_{1}$ и $F=F_{2}$, приходим к утверждению теоремы. 


\section{§ 6. Доказательство теоремы В}

Этот параграф посвящен получению нижней оценки величины $M(T+H)-$ $M(T)$.

ДокАЗАТЕЛЬСТво ТЕОРЕмЫ В. Пусть параметр $h$, точное значение которого будет выбрано далее, удовлетворяет условиям теоремы 2. Положим

$$
j_{1}(t ; h)=\int_{0}^{h}|S(t+u)| d u, \quad j_{2}(t ; h)=\left|\int_{0}^{h} S(t+u) d u\right|
$$

и обозначим буквой $E$ множество тех точек $t, T<t \leqslant T+H$, для которых $j_{1}(t ; h)>j_{2}(t ; h)$. Тогда на оставшейся части промежутка, которую мы обозначим $E_{1}$, выполнено равенство $j_{1}(t ; h)=j_{2}(t ; h)$. Положим $\nu_{0}=\left[H h^{-1}\right], H_{0}=h \nu_{0}$ и разобьем $\left(T, T+H_{0}\right]$ на $\nu_{0}$ промежутков $(T+(\nu-1) h, T+\nu h]=\left(T^{\prime}, T^{\prime}+h\right]$, $\nu=1,2, \ldots, \nu_{0}$. Пусть $\nu_{1}-$ количество таких промежутков, пересечение которых с множеством $E$ непусто. Занумеруем их в порядке возрастания правых концов и обозначим буквой $G$ объединение таких промежутков с четными номерами: $2,4, \ldots, 2 \nu_{2}=2\left[\nu_{1} / 2\right]$. Если точка $\tau$ множества $E$ принадлежит промежутку $\left(T^{\prime}, T^{\prime}+h\right]$ из $G$, то справедливо неравенство

$$
j_{1}(\tau ; h)=\int_{\tau}^{\tau+h}|S(t)| d t>\left|\int_{\tau}^{\tau+h} S(t) d t\right|=j_{2}(\tau ; h) .
$$

Это неравенство означает, что на интервале $(\tau, \tau+h)$ имеется точка перемены знака $S(t)$. Нетрудно убедиться в том, что эта точка содержится в интервале $\left(T^{\prime}, T^{\prime}+2 h\right)$. Поскольку расстояние между любыми двумя точками соседних промежутков из $G$ больше $h$, то никакие два из $\nu_{2}$ построенных таким образом интервалов $\left(T^{\prime}, T^{\prime}+2 h\right)$ не пересекаются. Отсюда следует, что на промежутке $\left(T, T+H_{0}\right]$ имеется по меньшей мере $\nu_{2}$ точек, в которых $S(t)$ меняет знак. Таким образом,

$$
M(T+H)-M(T) \geqslant M\left(T+H_{0}\right)-M(T) \geqslant \nu_{2} \geqslant \frac{\nu_{1}}{2}-1
$$

Так как множество $E$ целиком содержится в объединении $\nu_{1}$ промежутков длины $h$, то $\mu(E) \leqslant \nu_{1} h$. Поэтому число перемен знака $S(t)$ на $(T, T+H]$ оценивается снизу величиной

$$
\frac{\mu(E)}{2 h}-1
$$

Оценим теперь меру множества $E$ снизу. Пользуясь определением $E, E_{1}$ и неравенством Коши, находим

$$
\begin{aligned}
I_{1} & =\int_{T}^{T+H} j_{1}(t ; h) d t=\left(\int_{E_{1}}+\int_{E}\right) j_{1}(t ; h) d t \\
& =\int_{E_{1}} j_{2}(t ; h) d t+\int_{E} j_{1}(t ; h) d t \leqslant I_{2}+\sqrt{\mu(E)} \sqrt{I_{3}},
\end{aligned}
$$


где

$$
\begin{aligned}
& I_{2}=\int_{T}^{T+H} j_{2}(t ; h) d t=\int_{T}^{T+H}\left|S_{1}(t+h)-S_{1}(t)\right| d t, \\
& I_{3}=\int_{T}^{T+H}\left(\int_{0}^{h}|S(t+u)| d u\right)^{2} d t .
\end{aligned}
$$

После несложных преобразований получаем

$$
\mu(E) \geqslant \frac{\left(I_{1}-I_{2}\right)^{2}}{I_{3}} .
$$

Согласно теореме 2

$$
I_{1}=\int_{0}^{h} \int_{T+u}^{T+H+u}|S(t)| d t d u=\frac{H h}{\pi \sqrt{\pi}} \sqrt{\ln \ln T}\left(1+O(\ln \ln \ln X)^{-0.5}\right),
$$

так что

$$
I_{1}-I_{2} \geqslant \frac{H h}{\pi \sqrt{\pi}}\left(\sqrt{\ln \ln T}-\sqrt{\ln \left(h^{-1}\right)}\right)-c_{1} H h \sqrt{\frac{\ln \ln X}{\ln \ln \ln X}},
$$

где $c_{1}>0$ - достаточно большая постоянная. Положим теперь

$$
h=(\ln T)^{-1} \exp \left(\frac{40 c_{1} \ln \ln T}{\sqrt{\ln \ln \ln X}}\right) .
$$

Тогда

$$
\begin{aligned}
\sqrt{\ln \ln T}-\sqrt{\ln \left(h^{-1}\right)} & =\frac{\ln (h \ln T)}{\sqrt{\ln \ln T}+\sqrt{\ln \left(h^{-1}\right)}} \geqslant \frac{\ln (h \ln T)}{2 \sqrt{\ln \ln T}} \\
& \geqslant 20 c_{1} \sqrt{\frac{\ln \ln X}{\ln \ln \ln X}}
\end{aligned}
$$

и

$$
I_{1}-I_{2} \geqslant 0.1 H h 20 c_{1} \sqrt{\frac{\ln \ln X}{\ln \ln \ln X}}-c_{1} H h \sqrt{\frac{\ln \ln X}{\ln \ln \ln X}}=c_{1} H h \sqrt{\frac{\ln \ln X}{\ln \ln \ln X}} .
$$

Интеграл $I_{3}$ оценим, пользуясь теоремой 1 :

$$
\begin{aligned}
I_{3} & \leqslant h \int_{T}^{T+H} \int_{0}^{h}|S(t+u)|^{2} d u d t \leqslant h^{2} \int_{T}^{T+H+h}|S(t)|^{2} d t \\
& \leqslant h^{2}\left(\frac{H}{2 \pi^{2}} \ln \ln T+c H \sqrt{\ln \ln T}\right)<H h^{2} \ln \ln X .
\end{aligned}
$$

Таким образом,

$$
\mu(E) \geqslant\left(c_{1} H h \sqrt{\frac{\ln \ln X}{\ln \ln \ln X}}\right)^{2}\left(H h^{2} \ln \ln X\right)^{-1}=c_{1}^{2} H(\ln \ln \ln X)^{-1} .
$$

Переходя к оценке разности $M(T+H)-M(T)$, будем иметь

$$
M(T+H)-M(T) \geqslant c_{1}^{2} H h^{-1}(\ln \ln \ln X)^{-1}-1 \geqslant H(\ln T) \exp \left(-\frac{50 c_{1} \ln \ln X}{\sqrt{\ln \ln \ln X}}\right) .
$$

Теорема доказана. 


\section{Список литературы}

1. Selberg A. Contributions to the theory of the Riemann zeta-function // Archiv for Mathematik og Naturvidenskab. 1946. V. 48. № 5. P. 89-155.

2. Titchmarsh E. C. The zeros of the Riemann zeta-function // Proc. Royal Soc. 1935. V. 151. P. 234-255.

3. Титчмари Е. К. Теория дзета-функции Римана. М.: ИЛ, 1953.

4. Ghosh A. On Riemann's Zeta-Function - Sign Changes of $S(T) / /$ Recent Progress in Analytic Number Theory. V. 1. N. Y.: Academic Press, 1981. P. 29-46.

5. Карацуба А. А. О функции $S(t)$ // Изв. РАН. Сер. матем. 1996. Т. 60. № 5. С. 27-56.

6. Карацуба A.A. Плотностная теорема и поведение аргумента дзета-функции Римана // Матем. заметки. 1996. Т. 60. № 3. С. 448-449.

7. Королёв M. А. О числе перемен знака функции $S(t)$ на коротком промежутке // Докл. PAH. 2002. T. 382. № 4. C. 446-447.

8. Mueller $J$. On the Riemann zeta-function $\zeta(s)$ - gaps between sign changes of $S(t)$ // Mathematika. 1982. V. 29. № 2. P. 264-269.

9. Selberg A. Old and New Conjectures and Results About a Class of Dirichlet Series // Proc. of the Amalfi conference. Univ. Di Salerno, 1992. P. 365-387.

10. Карацуба A. А. Распределение нулей функции $\zeta\left(\frac{1}{2}+i t\right) / /$ Изв. АН СССР. Сер. матем. 1984. T. 48. № 6. C. 1214-1222.

11. Hardy G.H., Littlewood J.E. The zeros of Riemann's zeta-function on the critical line // Math. Zeitschr. 1921. V. 10. P. 283-317.

12. Hardy G. H., Littlewood J. E. The approximate functional equation in the theory of the zeta-function with applications to the divisor problem of Dirichlet and Piltz // Proc. London Math. Soc. 1922. V. 21. № 2. P. 39-74.

13. Карацуба А. А. Основы аналитической теории чисел. 1-е изд. М.: Наука, 1975.

14. Selberg $A$. On the remainder in the formula for $N(T)$, the number of zeros of $\zeta(s)$ in the strip $0<t<T / /$ Avhandlinger Norske Vid. Acad. Mat.-Nat. 1944. Kl. 1.

15. Постников А.Г. Эргодические вопросы теории сравнений и теории диофантовых приближений // Тр. матем. ин-та им. В.А. Стеклова АН СССР. 1966. Т. 82.

16. Кач M. Статистическая независимость в теории вероятностей, анализе и теории чисел. М.: ИЛ, 1963.

17. Ghosh $A$. On the Riemann zeta-function-mean value theorems and the distribution of $|S(t)|$ // J. of Number Theory. 1983. V. 17. P. 93-102.

18. Tsang K.-M. Some $\Omega$-theorems for the Riemann zeta-function // Acta Arithmetica. 1986. V. 46. № 4. P. 369-395. 